\title{
Birationally rigid complete intersections of quadrics and cubics
}

\author{
A.V.Pukhlikov \\ We prove birational superrigidity of generic Fano complete \\ intersections $V$ of type $2^{k_{1}} \cdot 3^{k_{2}}$ in the projective space $\mathbb{P}^{2 k_{1}+3 k_{2}}$, \\ under the condition that $k_{2} \geq 2$ and $k_{1}+2 k_{2}=\operatorname{dim} V \geq$ \\ 12, and of a few families of Fano complete intersections of \\ dimension 10 and 11. \\ Bibliography: 24 titles.
}

\section{Introduction}

0.1. Formulation of the main result. Let $k_{1}, k_{2} \in \mathbb{Z}_{+}$be a pair of nonnegative integers, $k_{1}+2 k_{2} \geq 4$. A smooth $\left(k_{1}+2 k_{2}\right)$-dimensional complete intersection

$$
V=F_{1} \cap \ldots \cap F_{k_{1}+k_{2}} \subset \mathbb{P}=\mathbb{P}^{2 k_{1}+3 k_{2}}
$$

of codimension $k=k_{1}+k_{2}$ in the projective space $\mathbb{P}$ over the field of complex numbers $\mathbb{C}$, where $F_{i} \subset \mathbb{P}$ are hypersurfaces of degree 2 for $i=1, \ldots, k_{1}$ and of degree 3 for $i=k_{1}+1, \ldots, k=k_{1}+k_{2}$ will be called a Fano complete intersection of the type $2^{k_{1}} \cdot 3^{k_{2}}$. Set $M=k_{1}+2 k_{2}=\operatorname{dim} V$. Obviously, $V$ is a Fano variety of index one, Pic $V=\mathbb{Z} H$ and $K_{V}=-H$, where $H$ is the class of a hyperplane section.

The main result of this paper is the following

Theorem 1. Assume that $k_{2} \geq 2$ and either $M=k_{1}+2 k_{2} \geq 12$, or the pair $\left(k_{1}, k_{2}\right)$ is one of the following five pairs:

$$
(5,3),(3,4),(1,5),(2,4),(0,5) \text {. }
$$

Then a generic (in the sense of the Zariski topology on the space of coefficients of the polynomials, defining the hypersurfaces $F_{1}, \ldots, F_{k_{1}+k_{2}}$ ) Fano complete intersection $V$ of type $2^{k_{1}} \cdot 3^{k_{2}}$ is birationally superrigid. In particular,

(i) there exists no rational dominant map $\gamma: V \rightarrow S$ onto a variety $S$ of positive dimension, the generic fibre of which $\gamma^{-1}(s)$ is of negative Kodaira dimension,

(ii) any birational map $\chi: V \rightarrow V^{\prime}$ onto a Fano variety $V^{\prime}$ with $\mathbb{Q}$-factorial terminal singularities and the Picard number rk Pic $V^{\prime}=1$ is a biregular isomorphism,

(iii) the group of birational automorphisms $\operatorname{Bir} V$ coincides with the group of biregular (projective) automorphisms Aut $V$ and for that reason is trivial. 
The properties (i)-(iii) follow immediately from the birational superrigidity, see [1]. In its turn, the birational superrigidity (understood in the sense of equality of the virtual and actual thresholds of canonical adjunction,

$$
c_{\mathrm{virt}}(\Sigma)=c(\Sigma)=n,
$$

for any mobile linear system $\Sigma \subset\left|-n K_{V}\right|$, see [1] for the definitions) follows immediately from the canonicity of every pair $\left(V, \frac{1}{n} \Sigma\right)$, where $\Sigma \subset\left|-n K_{V}\right|$ is a mobile system, or, equivalently, from the fact that the linear system $\Sigma$ has no maximal singularities.

Up to this day, birational superrigidity was shown for Fano complete intersections of index one $F_{1} \cap \ldots \cap F_{k}$ under the condition that for at least one degree the inequality $\operatorname{deg} F_{i} \geq 4$ holds [2, 3]. Theorem 1 states the superrigidity for complete intersections $V$ of quadrics and cubics under the assumption that $\operatorname{dim} V \geq 12$ and there are at least two cubics and also for certain five families of complete intersections of dimension 10 and 11. Thus the problem of birational superrigidity (of a generic variety) remains open for several families of Fano complete intersections of dimension $\leq 11$ and for the following three infinite series:

$$
2 \cdot 2 \cdot \ldots \cdot 2, \quad 2 \cdot 2 \cdot \ldots \cdot 2 \cdot 3 \text { and } 2 \cdot 2 \cdot \ldots \cdot 2 \cdot 4
$$

in the arbitrary dimension. Those varieties require further improvement of the techniques of the proof.

In the next subsection we give a precise meaning to the condition of general position of the variety $V$ in its family. The main difficulty in the proof of Theorem 1 is to show that this condition does realize, that is, there is a non-empty Zarisky open subset in the space of parameters, corresponding to the varieties $V$, satisfying that condition. The very proof of birational superrigidity take a few pages $(\S 1)$, almost all paper is devoted to the proof of the conditions of general position.

In Sec. 0.3 we give a more detailed description of the structure of the paper, of the main idea of the proof of Theorem 1 and of the position of that theorem among the other results on birational rigidity.

0.2. Conditions of general position. As usual, the conditions of general position (or the regularity conditions) are of local nature and should be satisfied at every point of the variety $V$. Let $o \in V \subset \mathbb{P}$ be some point, $\mathbb{C}^{M+k} \subset \mathbb{P}$ a standard affine set with the coordinates $\left(z_{1}, \ldots, z_{M+k}\right)$, where $o=(0, \ldots, 0)$. Let

$$
f_{i}=q_{i, 1}+q_{i, 2}
$$

for $i=1, \ldots, k_{1}$, be the equations of the quadrics $F_{1}, \ldots, F_{k_{1}}$ and

$$
f_{i}=q_{i, 1}+q_{i, 2}+q_{i, 3}
$$

for $i=k_{1}+1, \ldots, k_{1}+k_{2}$, the equations of the cubics $F_{k_{1}+1}, \ldots, F_{k}$, decomposed into homogeneous in $z_{*}$ components. The tangent space $T_{o} V \subset \mathbb{C}^{M+k}$ is given be the system of equations

$$
q_{1,1}=\ldots=q_{k, 1}=0 .
$$


We denote its projectivization $\mathbb{P}\left(T_{o} V\right) \cong \mathbb{P}^{M-1}$ by the symbol $\mathbb{T}$. Set $\bar{q}_{i, j}=\left.q_{i, j}\right|_{\mathbb{T}}$. Let us introduce the following sets of pairs of indices:

$$
J_{1}=\{(i, 2) \mid 1 \leq i \leq k\}, \quad J_{2}=\left\{(i, 3) \mid k_{1}+1 \leq i \leq k_{1}+k_{2}\right\}
$$

and $J=J_{1} \cup J_{2}$. The first (traditional) regularity condition is formulated in the following way:

(R1) The system of homogeneous equations

$$
\left\{\bar{q}_{i, 2}=0 \mid 1 \leq i \leq k\right\}
$$

defines in $\mathbb{T}$ a closed subset of pure codimension $k$, whereas the system of equations

$$
\left\{\bar{q}_{i, j}=0 \mid 1(i, j) \in J\right\}
$$

defines in $\mathbb{T}$ either the empty set or a finite set of points, linearly independent in $\mathbb{T}$.

The last condition (on the linear independence) is a new addition to the usual regularity condition [2, 3], which requires only that the set (2) should be finite. It is clear that if the condition (R1) is satisfied at the point $o$, then on $V$ lie at most $M$ lines, passing through that point. The second regularity condition was introduced in [3].

(R2) None of the irreducible components of the closed algebraic set (1) is contained in a linear subspace of codimension two in $\mathbb{T}$.

In [3] the condition (R2) was called correctness in the quadratic terms.

To formulate the third regularity condition, we need some additional constructions. Assume that the set (2) is non-empty and denote it by the symbol $\Delta$. Let

$$
\Sigma_{1}^{\mathbb{T}}=<\bar{q}_{i, 2} \mid 1 \leq i \leq k>
$$

be the linear system of quadrics on $\mathbb{T}$, generated by $\bar{q}_{i, 2}$ and $\Sigma_{2}^{\mathbb{T}}$ the linear system of cubics, consisting of all linear combinations of the form

$$
\sum_{i=1}^{k} \bar{s}_{i}(z) \bar{q}_{i, 2}+\sum_{i=k_{1}+1}^{k_{1}+k_{2}} \lambda_{i} \bar{q}_{i, 3},
$$

where $\lambda_{i}$ are constants and $\bar{s}_{i}(z)=\left.s_{i}(z)\right|_{\mathbb{T}}$ are linear polynomials on $\mathbb{T}$. Let

$$
\mathcal{P}=\underbrace{\sum_{1}^{\mathbb{T}} \times \ldots \times \Sigma_{1}^{\mathbb{T}}}_{k-2} \times \underbrace{\sum_{2}^{\mathbb{T}} \times \ldots \times \Sigma_{2}^{\mathbb{T}}}_{k_{2}-2}
$$

be the space of all tuples of polynomials $\left(g_{1}, \ldots, g_{M-4}\right)$, where the first $k-2$ polynomials are taken from the space $\Sigma_{1}^{\mathbb{T}}$, and the last $k_{2}-2$ from the space $\Sigma_{2}^{\mathbb{T}}$. Now let us formulate the third and last regularity condition:

(R3) for any irreducible subvariety $R \subset \mathbb{T}$ of degree $d_{R} \geq 1$ and codimension 3 there exists a non-empty Zarisky open subset $U_{R} \subset \mathcal{P}$, such that for any tuple

$$
\left(g_{1}, \ldots, g_{M-4}\right) \in U_{R}
$$


the following inequality holds:

$$
\sum_{p \in \Delta} \operatorname{dim} \mathcal{O}_{p, R} /\left(g_{1}, \ldots, g_{M-4}\right) \leq 2^{k-4} 3^{k_{2}-1} d_{R}
$$

Note that by the condition $(\mathrm{R} 1)$ for a generic tuple $\left(g_{1}, \ldots, g_{M-4}\right) \in \mathcal{P}$ the scheme

$$
\left(R \circ\left\{g_{1}=0\right\} \circ \ldots \circ\left\{g_{M-4}=0\right\}\right)
$$

is zero-dimensional and for that reason is of degree $2^{k-2} 3^{k_{2}-2} d_{R}$. The condition (R3) means that at most $3 / 4$ of that full degree is concentrated at the points of the set $\Delta$. This is a very strong condition, since it should be satisfied for an arbitrary subvariety $R$ of the projective space $\mathbb{T}$.

Let

$$
\mathcal{F}_{s m} \subset \prod_{i=1}^{k_{1}} H^{0}\left(\mathbb{P}, \mathcal{O}_{\mathbb{P}}(2)\right) \times \prod_{i=k_{1}+1}^{k} H^{0}\left(\mathbb{P}, \mathcal{O}_{\mathbb{P}}(3)\right)
$$

be the space of tuples $\left(f_{1}, \ldots, f_{k}\right)$, defining a smooth complete intersection of the type $2^{k_{1}} 3^{k_{2}}$ in $\mathbb{P}$.

Theorem 2. There exists a non-empty Zarisky open subset $\mathcal{F}_{\text {reg }} \subset \mathcal{F}_{\mathrm{sm}}$ of tuples $\left(f_{1}, \ldots, f_{k}\right)$, for which the corresponding complete intersection $V\left(f_{1}, \ldots, f_{k}\right)=\left\{f_{1}=\right.$ $\left.\ldots=f_{k}=0\right\} \subset \mathbb{P}$ satisfies the regularity conditions (R1)-(R3) at every point $o \in V$.

Now we can make the main result of the present paper more precise.

Theorem 3. Let $V$ be a Fano complete intersection of the type $2^{k_{1}} 3^{k_{2}}$ in $\mathbb{P}$, satisfying the regularity conditions (R1)-(R3) at every point $o \in V$, where the numbers $k_{1}, k_{2}$ satisfy the assumptions of Theorem 1 . Then the variety $V$ is birationally superrigid. In particular, the claims (i)-(iii) of Theorem 1 hold.

Obviously, Theorem 1 follows from Theorems 2 and 3, which are independent of each other and will be shown separately.

0.3. The structure of the paper and historical remarks. Theorem 1 is proved in $\S 1$. Its proof takes a few pages and makes use of the technique of hypertangent linear system and the $8 n^{2}$-inequality. In two words, the idea of the proof can be explained as follows. In the preceding papers (see [1-5] and the bibliography in [1]) to show birational superrigidity of Fano complete intersections, one constructed, starting from the self-intersection $Z$ of a movable linear system $\Sigma$ on $V$ with a maximal singularity, an effective1-cycle $Y \subset V$, satisfying the inequality

$$
\text { mult }_{o} Y>\operatorname{deg} Y
$$

which is, of course, impossible, and brings the assumption that such a system $\Sigma$ exists to a contradiction, thus proving birational superrigidity of the variety $V$. For complete intersections of quadrics and cubics this construction does not work in the straightforward way: the technique of hypertangent systems makes it possible to construct a curve $Y \subset V$ that has a high multiplicity mult ${ }_{o} Y$, which, however, does not exceed its degree $\operatorname{deg} Y$, so that there is no contradiction. 
An easy analysis shows that the irreducible curve $C \subset V$, which is not a line (that is, a curve of degree $\operatorname{deg} C \geq 2$ ), satisfies an essentially stronger inequality than the inequality, opposite to (4): the estimate

$$
\text { mult }_{o} C \leq \frac{2}{3} \operatorname{deg} C
$$

holds. Breaking the effective 1-cycle $Y$ into two sub-cycles:

$$
Y=Y_{1}+Y_{\geq 2}
$$

where $Y_{1}$ is concentrated on the lines, passing through the point $o$, and $Y_{\geq 2}$ is concentrated on the curves of degree $\geq 2$, we see that the strategy of the proof, described above, would be successful if the sub-cycle $Y_{1}$ is not too large, that is, if the ratio $\operatorname{deg} Y_{1} / \operatorname{deg} Y$ is small enough. Using the more precise estimate (5) for the sub-cycle $Y_{\geq 2}$, one can get a contradiction and prove birational rigidity. This is possible, provided that the "outcome" of the technique of hypertangent divisors, applied to the self-intersection $Z$ of the linear system $\Sigma$ (or to a certain irreducible component of that self-intersection), is an effective 1-cycle $Y$, containing "not too many lines". Proof of Theorem 3, realizing the strategy, described above, is given in $\S 1$.

The main part of the paper, $\S \S 2-5$, is about estimating the number of lines (taking into account the multiplicities), emerging as the result of intersecting an arbitrary irreducible subvariety with hypertangent divisors. In $\S \S 2-4$ we consider the local problem: to estimate the multiplicity of a generic tuple of polynomials from a given linear system with an arbitrary effective cycle at a fixed point. In $\S 5$ the problem is globalized: we estimate the sum of local intersection multiplicities over the base points of the linear system, and on that basis prove Theorem 2 .

The theory of birational rigidity of Fano complete intersections has a long history, starting from the work of Fano himself [6], where he studied complete intersections $V_{2 \cdot 3} \subset \mathbb{P}^{5}$ of a quadric and a cubic and complete intersections of three quadrics $V_{2 \cdot 2 \cdot 2} \subset \mathbb{P}^{6}$. Fano gave a description (as it turned out later, non-complete [7]) of generators of the group of birational self-maps $\operatorname{Bir} V_{2 \cdot 3}$ and formulated the theorem on non-rationality for the both classes of varieties. As it was discovered later [8], his arguments contained numerous mistakes and gaps and can not be considered even as a first approximation to the rigorous proof. At the same time, in his works Fano outlined many important ideas and constructions which later were proved essential in birational geometry (such as the "double projection" or the NoetherFano inequality).

In 1970 in their pioneer paper [8] V.A.Iskovskikh and Yu.I.Manin gave the first ever rigorous proof of birational superrigidity (in the modern terminology) for one class of three-dimensional Fano varieties, the smooth three-dimensional quartics $V_{4} \subset \mathbb{P}^{4}$. With a minimal modification (which deal with the easier part of the proof, that on exclusion or untwisting maximal curves lying on the variety itself) the proof of V.A.Iskovskikh and Yu.I.Manin worked for the Fano double spaces $V_{2} \stackrel{2: 1}{\rightarrow} \mathbb{P}^{3}$ of 
index 1 and the double quadrics $V_{4} \stackrel{2: 1}{\rightarrow} Q_{2} \subset \mathbb{P}^{4}$ of index 1, see [7]. However, the proof of birational rigidity and description of the group of birational self-maps of the variety $V_{2 \cdot 3}$ (announced in [9]) turned out to be a much harder problem. The test class method, developed in [8], worked successfully for varieties of degree at most 4 only, and already the degree $\operatorname{deg} V_{2 \cdot 3}=6$ made a serious obstruction. The arguments of [7], used for the exclusion of the infinitely near maximal singularity [7, Sec. 4.8, Step 3], were erroneous, and this, most essential step of the proof remained an open problem until 1987 [10]. A complete proof of the theorem on birational rigidity of a generic complete intersection of a quadric and a cubic of dimension three see in [11]. The problem of description of the structures of a rationally connected fibre space and the group of birational self-maps of the variety $V_{2 \cdot 2 \cdot 2}$ is still open. (Note that birational geometry of Fano complete intersections was also studied by means of the transcendental method; there are not many such papers, see [12, 13].)

The work on extending the test class method into arbitrary dimension was started in [14, 15]. The ideas developed in [14] are sufficient for the proof of birational superrigidity of generic complete intersections of a quadric and a quartic $V_{2 \cdot 4} \subset \mathbb{P}^{6}$ of dimension 4 and index 1 . This result was announced in 1985, but its complete proof was not published. (Later in [16] birational superrigidity of complete intersections of a quadric and a quartic, not containing planes, was shown via the $8 n^{2}$-inequality, however, the proof of that inequality that was known at the time turned out to be erroneous and a complete proof was obtained later, see the history of this problem in [17].)

Replacing the test class method by the method of counting multiplicities, introducing the techniques of hypertangent divisors and some other ideas [4] made it possible to prove birational superrigidity of Fano hypersurfaces of index 1 and later of generic complete intersections $V_{d_{1} \cdot \ldots \cdot d_{k}} \subset \mathbb{P}^{M+k}$ of index 1 for $M \geq 2 k+1$, $\operatorname{dim} V \geq 4$ [2]. The next step in the development of this area was systematic use of the connectedness principle of Shokurov and Kollár [18] and the proof of the $8 n^{2}$-inequality, which formed a basis for proving birational superrigidity for a wider class of complete intersections with $M \geq k+3, d_{k} \geq 4[3$. The latter paper is the immediate predecessor of the present one: here we remove the restriction $d_{k} \geq 4$. As we noted above, now the problem of birational (super)rigidity remains open for very few classes of Fano complete intersections of index 1. The conjecture on bitational superrigidity of arbitrary smooth Fano complete intersections of index 1 was formulated by the author many times (see, for instance, [19]).

The method of maximal singularities makes it also possible to prove birational superrigidity of the varieties fibred into Fano complete intersections over the projective line $\mathbb{P}^{1}$, see [20, 21], but this is a different topic.

The work on the problem, a solution of which makes the contents of the present paper, was completed in spring 2012. As far as the author knew, the problem of estimating the multiplicity for a generic tuple of polynomials in a subvariety of a given codimension has never been considered before. However, in June 2012 A.G.Khovanskii informed the author that a different, but close problem, that of 
estimating the multiplicity of an isolated solution of a system of $n$ equations

$$
\phi_{1}=\ldots=\phi_{n}=0
$$

in $n$ complex variables for a tuple $\left(\phi_{1}, \ldots, \phi_{n}\right)$ in a ring of Noetherian functions $K \supset$ $\mathbb{C}\left[z_{1}, \ldots, z_{n}\right]$, finitely generated over $\mathbb{C}\left[z_{*}\right]$ and closed with respect to differentiations, was studied in 22. In the latter paper the estimate was obtained in terms of invariants of the ring $K$ using the methods that were essentially different from the algebro-geometric techniques used in $\S \S 2-4$ : by reduction to the one-dimensional problem of restricting a polynomial onto a trajectory of a polynomial vector field, via estimating the complexity of an integral manifold of an analytic vector-function and using deformations. In our paper we consider a less general (and in fact somewhat different) problem, but a considerably stronger and (which is especially important for applications to birational geometry) effective estimate of the multiplicity, which is not implied by the estimates of [22].

The method of solution, developed in this paper (that is, the method of the proof of Theorem 2), is new. A simplified version of this method (for a system of $N$ polynomial equations in $N$ variables, without involving an effective cycle $R$ of the given degree) was published in [23].

The very fact that the problem of estimating multiplicity of an (isolated) solution of a system of $n$ equations in $n$ variables emerges in different contexts, requires different types of techniques and has various applications, is remarkable; the author is grateful to A.G.Khovanskii for pointing out the paper [22].

\section{§1. Proof of birational superrigidity}

In this section we prove Theorem 3.

1.1. Start of the proof. The $8 n^{2}$-inequality. The proof of Theorem 3 starts in the standard way. Let us fix a complete intersection $V \subset \mathbb{P}$, satisfying the assumptions of the theorem, and a mobile linear system $\Sigma \subset|n H|$ (where, let us remind the reader, $H$ is the class of a hyperplane section of the variety $V$ ) with a maximal singularity [1]. The latter means that for some birational morphism $\varphi: V^{\sharp} \rightarrow V$, where $V^{\sharp}$ is a non-singular projective variety, and an exceptional divisor $E^{\sharp} \subset V^{\sharp}$ (the maximal singularity) the Noether-Fano inequality holds

$$
\operatorname{ord}_{E^{\sharp}} \varphi^{*} \Sigma>n a\left(E^{\sharp}, V\right) .
$$

The irreducible subvariety $B=\varphi\left(E^{\sharp}\right) \subset V$ is called the centre of the maximal singularity $E^{\sharp}$. The inequality $\operatorname{mult}_{B} \Sigma>n$ holds. There are three options for the codimension of the subvariety $B$ :

(i) $\operatorname{codim} B=2$,

(ii) $\operatorname{codim} B=3$,

(iii) $\operatorname{codim} B \geq 4$. 
As it was shown in, for example, [2] (that argument can be found in many papers on birational rigidity), the first option does not realize, since for the numerical Chow group we have $A^{2} V=\mathbb{Z} H^{2}$. The second option is excluded, for instance, in [3]. Thus we will assume that $\operatorname{codim} B \geq 4$.

At first our arguments repeat the proof of Theorem 3 in [3, Sec. 3] almost word for word. We assume that the codimension of the subvariety $B$ is minimal among all centres of maximal singularities of the system $\Sigma$; in particular, $B$ is not strictly contained in the centre of another maximal singularity, if there are any. Take a point of general position $o \in B$. Let $\lambda: V^{+} \rightarrow V$ be its blow up, $E=\lambda^{-1}(o) \cong \mathbb{P}^{M-1}$ the exceptional divisor, $\Sigma^{+}$the strict transform of the mobile system $\Sigma$ on $V^{+}$, $Z=\left(D_{1} \circ D_{2}\right)$ and $Z^{+}$are the self-intersection of the system $\Sigma$ and its strict transform on $V^{+}$, respectively.

Proposition 1.1 (the $8 n^{2}$-inequality). There exist a linear subspace $P \subset E$ of codimension two, satisfying the inequality

$$
\operatorname{mult}_{o} Z+\operatorname{mult}_{P} Z^{+}>8 n^{2}
$$

If mult $_{o} Z \leq 8 n^{2}$, then the linear subspace $P$ is uniquely determined by the system $\Sigma$.

Proof was given in [17, Sec. 4.1].

Let $\Lambda \supset P$ be a generic hyperplane in $E \cong \mathbb{P}^{M-1}$, containing the subspace $P, L \in|H|$ a generic hyperplane section, containing the point $o$ and such that $L^{+} \cap E=\Lambda$, where $L^{+}$is the strict transform of the divisor $L$ on $V^{+}$. By genericity of the choice of $\Lambda$ and $L$ none of the irreducible components of the cycle $Z$ is contained in $L$, so that the scheme-theoretic intersection $Z_{L}=(Z \circ L)$ is well defined. The effective cycle $Z_{L}$ of codimension three satisfies the inequality

$$
\text { mult }_{o} Z_{L} \geq \text { mult }_{o} Z+\operatorname{mult}_{P} Z^{+}>8 n^{2}
$$

and the equality $\operatorname{deg} Z_{L}=\operatorname{deg} Z=d n^{2}$, where $d=2^{k_{1}} 3^{k_{2}}$ is the degree of the complete intersection $V$. Therefore, there exists an irreducibel subvariety $Q \subset V$ of codimension three (an irreducible component of the cycle $Z_{L}$ ), satisfying the estimate

$$
\frac{\operatorname{mult}_{o} Q}{\operatorname{deg} Q}>\frac{8}{d}
$$

Let $Q_{E}=\left(Q^{+} \circ E\right)=\sum_{i \in I} m_{i} R_{i}$ be the projectivized tangent cone to the subvariety $Q$ at the point $o$ (here $Q^{+} \subset V^{+}$is the strict transform of the subvariety $Q$, the subvarieties $R_{i} \subset E$ are irreducible components of the effective cycle $Q_{E}, m_{i} \geq 1$; here and everywhere in this paper constructions of elementary intersection theory are understood in the sense of [24]). Now let us apply to the subvariety $Q \subset V$ the technique of hypertangent divisors in the way in which it was done in [3], but with a small modification. Namely, at each step when intersecting with a (hyper)tangent divisor, we will ignore only the emerging irreducible components that do not contain the point $o$. 


\subsection{The technique of hypertangent divisors. Let}

$$
\Sigma_{1}=\left\{\left.\lambda_{1} q_{1,1}\right|_{V}+\ldots+\left.\lambda_{k} q_{k, 1}\right|_{V}\right\}
$$

be the $k$-dimensional space of equations of tangent hyperplanes at the point $o$. By the condition (R1) for any non-zero tuple $\left(\lambda_{1}, \ldots, \lambda_{k}\right)$ the corresponding tangent divisor

$$
T\left(\lambda_{*}\right)=\left\{\left.\lambda_{1} q_{1,1}\right|_{V}+\ldots+\left.\lambda_{k} q_{k, 1}\right|_{V}=0\right\}
$$

satisfies the equality

$$
T^{E}\left(\lambda_{*}\right)=\left(T^{+}\left(\lambda_{*}\right) \circ E\right)=\left\{\lambda_{1} \bar{q}_{1,2}+\ldots+\lambda_{k} \bar{q}_{k, 2}=0\right\} .
$$

In particular, set $T_{i}=\left\{\left.q_{i, 1}\right|_{V}=0\right\}$. Since none of the irreducible components of the effective cycle $\left(T_{1}^{E} \circ \ldots \circ T_{k}^{E}\right)$ of codimension $k$ by the condition (R2) is contained in the linear subspace of codimension two in $E$, we get the equality

$$
\operatorname{codim}_{E}\left(T_{1}^{E} \cap \ldots \cap T_{k}^{E} \cap \Lambda\right)=k+1 .
$$

The support of the cycle $Q_{E}$, constructed above, is contained in the hyperplane $\Lambda$. By the equality (7), for $(k-2)$ generic divisors $D_{1,1}, \ldots, D_{1, k-2}$ in the linear system $\Sigma_{1}$ the equality

$$
\operatorname{codim}_{E}\left(Q_{E} \cap D_{1,1}^{E} \cap \ldots \cap D_{1, k-2}^{E}\right)=k+1
$$

holds, where $D_{1, i}^{E}=\left(D_{1, i}^{+} \circ E\right)$. Therefore, in a neighborhood of the point $o$ we also have

$$
\operatorname{codim}_{o}\left(Q \cap D_{1,1} \cap \ldots \cap D_{1, k-2}\right)=k+1 .
$$

Now let us construct the following sequence of effective cycles $Q_{i}$, where $i=0,1 \ldots, k-$ 2:

(i) $Q_{0}=Q$,

(ii) $Q_{i+1}$ is obtained by removing from the effective cycle $\left(Q_{i} \circ D_{1, i+1}\right)$ all irreducible components, not containing the point $o$.

This procedure is well defined: since all components of each of the cycles $Q_{i}$ contain the point $o$, by the equality (8) none of the components of the cycle $Q_{i}$ is contained in the divisor $D_{1, i+1}$. In particular, $\operatorname{codim} Q_{i}=i+3$ for $i=0, \ldots, k-2$. The degree $\operatorname{deg} Q_{i}$ is not increasing (in fact, it is decreasing, if some components are indeed removed in the process of this construction), so that $\operatorname{deg} Q_{k-2} \leq \operatorname{deg} Q$, whereas for the multiplicity at the point $o$ we get the equality

$$
\text { mult }_{o} Q_{k-2}=2^{k-2} \text { mult }_{o} Q .
$$

Now let us consider the hypertangent linear system

$$
\Sigma_{2}=\left\{\left.h(z)\right|_{V}=\left.\sum_{i=1}^{k} s_{i}(z) q_{i, 1}\right|_{V}+\left.\sum_{i=k_{1}+1}^{k_{1}+k_{2}} \lambda_{i}\left(q_{i, 1}+q_{i, 2}\right)\right|_{V}\right\} .
$$


Obviously, $\Sigma_{2}^{+} \subset|2 H-3 E|$ : the projectivized tangent cone of the divisor $\left.h\right|_{V}=0$ at the point $O$ is given by the equation

$$
-\left(\sum_{i=1}^{k} \bar{s}_{i} \bar{q}_{i, 2}+\sum_{i=k_{1}+1}^{k_{1}+k_{2}} \lambda_{i} \bar{q}_{i, 3}\right) \in \Sigma_{2}^{\mathbb{T}} .
$$

Let $\left(D_{2,1}, \ldots, D_{2, k_{2}-2}\right) \in \Sigma_{2}^{\times\left(k_{2}-2\right)}$ be a generic tuple of $\left(k_{2}-2\right)$ hypertangent divisors. By the condition (R1) the closed set

$$
Q_{E} \cap\left(\bigcap_{i=1}^{k-2} D_{1, i}^{E}\right) \bigcap\left(\bigcap_{i=1}^{k_{2}-2} D_{2, i}^{E}\right)
$$

is zero-dimensional, so that the closed set

$$
Q \cap\left(\bigcap_{i=1}^{k-2} D_{1, i}\right) \cap\left(\bigcap_{i=1}^{k_{2}-2} D_{2, i}\right)
$$

is one-dimensional in a neighborhood of the point $o$. Let us continue to construct the chain of effective cycles $Q_{i}, i=k-2, \ldots, M-4$, where $Q_{k-2}$ are already constructed and $Q_{i+1}$ is obtained by removing from the effective cycle $\left(Q_{i} \circ D_{2, i+3-k}\right)$ all irreducible components, not containing the point $o$.

Set $C=Q_{M-4}$. This is a 1-cycle, each component of which contains the point $o$. We have $\operatorname{deg} C \leq 2^{k_{2}-2} \operatorname{deg} Q$, and for the multiplicity at the point $o$ the equality

$$
\text { mult }_{o} C=2^{k-2} 3^{k_{2}-2} \text { mult }_{o} Q
$$

holds. Taking into account the choice of the subvariety $Q$, we get

$$
\frac{\text { mult }_{o} C}{\operatorname{deg} C}>\frac{2^{k-2} 3^{k_{2}-2}}{2^{k_{2}-2}} \cdot \frac{8}{d}=\frac{8}{9} .
$$

This estimate is obviously very strong, however it still does not allow to get a contradiction: any line, passing through the point $o$, satisfies this inequality. Now let us use the condition (R3), bounding the input of the lines into the effective cycle C.

1.3. The effective 1-cycle, free from lines. Now let us write down $C=$ $C_{1}+C_{>2}$, where the support of the effective 1-cycle $C_{1}$ consists of lines, whereas the support of the effective 1-cycle $C_{\geq 2}$ consists of the curves of degree $\geq 2$. Obviously, mult $_{o} C_{1}=\operatorname{deg} C_{1}$.

Lemma 1.1. For any irreducible curve $\Gamma \subset V$ of degree $\operatorname{deg} \Gamma \geq 2$ the following inequality holds:

$$
\operatorname{mult}_{o} \Gamma \leq \frac{2}{3} \operatorname{deg} \Gamma .
$$


Proof. By the condition (R1), the system of homogeneous equations in the space $\mathbb{C}^{M+k}$

$$
q_{*, *}(z)=0,
$$

consisting of all irreducible components of all $k$ equations $f_{i}$, defines either the origin $o \in \mathbb{C}^{M+k}$, or a finite set of lines, passing through the point $o$. Obviously, a line $t\left(a_{1}, \ldots, a_{M+k}\right)$ lies on $V$ if and only if $q_{i, j}\left(a_{*}\right)=0$ for all $i, j$. Assume that the curve $\Gamma \ni o$ is not a line and satisfies the inequality

$$
\operatorname{mult}_{o} \Gamma>\frac{2}{3} \operatorname{deg} \Gamma \text {. }
$$

It is clear that $\Gamma$ is contained in the support of any tangent divisor $D \in \Sigma_{1}$ (that is, of such a divisor that $D^{+} \in|H-2 E|$ ) and hypertangent divisor $D \in \Sigma_{2}$ (that is, $\left.D^{+} \in|2 H-3 E|\right)$. Therefore, on the curve $\Gamma$ the following polynomials vanish identically:

$$
q_{i, 1}, \quad i=1, \ldots, k
$$

and

$$
q_{i, 1}+q_{i, 2}, \quad i=k_{1}+1, \ldots, k .
$$

Besides, since $\Gamma \subset V$, on that curve identically vanish the polynomials

$$
f_{i}=q_{i, 1}+q_{i, 2}, \quad i=1, \ldots, k_{1},
$$

and

$$
f_{i}=q_{i, 1}+q_{i, 2}+q_{i, 3}, \quad i=k_{1}+1, \ldots, k .
$$

We conclude that all homogeneous polynomials $q_{i, j}$ vanish on $\Gamma$. But then $\Gamma$ is a line. This contradiction proves the lemma.

Corollary 1.1. The following estimate holds:

$$
\text { mult }_{o} C_{\geq 2} \leq \frac{2}{3} \operatorname{deg} C_{\geq 2}
$$

To complete the proof of Theorem 3, it remains to estimate from above the input of lines into the 1 -cycle $C$, that is, the ratio $\operatorname{deg} C_{1} / \operatorname{deg} C$.

Let us consider generic tangent divisors $D_{1, i}$ that were used to construct the curve $C$. If

$$
\left.\left(\lambda_{1, i} q_{1,1}+\lambda_{2, i} q_{2,1}+\ldots+\lambda_{k, i} q_{k, 1}\right)\right|_{V}
$$

is the equation of the divisor $D_{1, i}$, then obviously

$$
g_{i}=-\left(\lambda_{1, i} \bar{q}_{1,2}+\lambda_{2, i} \bar{q}_{2,2}+\ldots+\lambda_{k, i} \bar{q}_{k, 2}\right) \in \Sigma_{1}^{\mathbb{T}}
$$

is the equation of its projectivized tangent cone. Therefore, $\left(g_{1}, \ldots, g_{k-2}\right)$ form a generic tuple, that is, generic element of the space $\left(\Sigma_{1}^{\mathbb{T}}\right)^{\times(k-2)}$. In a similar way, the projectivized tangent cone of the divisor $D_{2, i}, i=1, \ldots, k_{2}-2$, has the equation

$$
g_{k-2+i}=\sum_{j=1}^{k} \bar{s}_{j} \bar{q}_{j, 2}+\sum_{j=k_{1}+1}^{k_{1}+k_{2}} \lambda_{j} \bar{q}_{j, 3} \in \Sigma_{2}^{\mathbb{T}}
$$


(to simplify our notations, we omit in the right hand side of this equality the second index for $\bar{s}_{j}$ and $\lambda_{j}$ that indicates the dependence on the number $i$ of the divisor $D_{2, i}$ ), whereas $\left(g_{1}, \ldots, g_{M-4}\right) \in \mathcal{P}$ is a generic element of the space $\mathcal{P}$. Since at each step of the construction of the curve $C$ the intersection with the (hyper)tangent divisor $D_{i, j}$ is proper, the zero-dimensional cycle $C_{E}=\left(C^{+} \circ E\right)$ is the scheme-theoretic intersection

$$
\left(Q_{E} \circ D_{1,1} \circ \ldots \circ D_{1, k-2} \circ D_{2,1} \circ \ldots \circ D_{2, k_{2}-2}\right),
$$

that is, we get the equality of 0 -cycles

$$
\left(C_{1}^{+} \circ E\right)+\left(C_{\geq 2}^{+} \circ E\right)=\sum_{i \in I} m_{i}\left(R_{i} \circ D_{1,1} \circ \ldots \circ D_{2, k_{2}-2}\right)
$$

in the right hand side we get the scheme-theoretic intersection with all $M-4$ (hyper)tangent divisors $D_{i, j}$ that took part in the procedure of constructing the 1cycle $C$. For any line $L \subset V$, passing through the point $o$, in the notations of Sec. 0.2 we have

$$
\left(L^{+} \cap E\right) \in \Delta
$$

(the set $\Delta$ consists precisely of all tangent directions of all lines on $V$, passing through the point $o)$. Therefore, the support of the 0 -cycle $\left(C_{1}^{+} \circ E\right)$ is a subset of the finite set $\Delta$. By the condition (R3), that is, by the estimate (3),

$$
\sum_{p \in \Delta} \sum_{i \in I} m_{i} \operatorname{dim} \mathcal{O}_{p, R_{i}} /\left(g_{1}, \ldots, g_{M-4}\right) \leq 2^{k-4} 3^{k_{2}-1} \operatorname{mult}_{o} Q
$$

since, obviously, the degree of the effective cycle $Q_{E}$ of codimension three on $E$ is precisely mult $_{\mathrm{o}} Q$. The more so,

$$
\delta=\operatorname{deg}\left(C_{1}^{+} \circ E\right)=\operatorname{deg} C_{1} \leq 2^{k-4} 3^{k_{2}-1} \text { mult }_{o} Q .
$$

Therefore, for the 1-cycle $C_{\geq 2}$ the equality

$$
\text { mult }_{o} C_{\geq 2}=2^{k-2} 3^{k_{2}-3} \text { mult }_{o} Q-\delta
$$

and the inequality

$$
\operatorname{deg} C_{\geq 2} \leq 2^{k_{2}-2} \operatorname{deg} Q-\delta
$$

hold. By easy computations from the inequality of Corollary 1.1 we get the estimate

$$
\delta \geq 2^{k-2} 3^{k_{2}-1} \text { mult }_{o} Q-2^{k_{2}-1} \operatorname{deg} Q .
$$

Combining the last inequality with (9), we obtain finally

$$
2^{k_{2}-1} \operatorname{deg} Q \geq 2^{k-4} 3^{k_{2}} \text { mult }_{o} Q .
$$

Recalling that $k=k_{1}+k_{2}$ and $d=2^{k_{1}} 3^{k_{2}}$, we obtain from there the inequality

$$
\text { mult }_{o} Q \leq \frac{8}{d} \operatorname{deg} Q
$$


which contradicts the inequality (6). This contradiction excludes the third (and last) option codim $B \geq 4$ and completes the proof of Theorem 3 .

Remark 1.1. In all previous papers (see, for instance, [1, 4, 5, 19]) the technique of hypertangent divisors was used in a somewhat different way: at each step when intersecting with a hypertangent divisor, we selected an irreducibe component with the maximal ratio mult $/ \mathrm{deg}$, the other components of the scheme-theoretic intersection were ignored. In the argument given above, in order to estimate the input of lines at the last step, we needed to control the whole cycle of intersection, not only one of its components. This type of arguments works for the previous problems, too: instead of selecting the component with the maximal ratio mult $/ \mathrm{deg}$, one may consider the whole cycle of the scheme-theoretic intersection, removing only the components that do not contain the point $o$ (since the regularity conditions ensure that the procedure of taking the intersection with a hypertangent divisor is well defined in a neighborhood of that point only).

\section{§2. The local multiplicities. I. The spaces of tuples of polynomials}

In this section we give a precise formulation of the problem of estimating the local multiplicity: introduce the space of tuples of polynomials, define the local effective multiplicities of intersection and formulate the main result (Theorem 4). The theory developed in this and two subsequent sections is independent of $\S 1$ and is self-contained. The notations are also independent of $\S 1$.

2.1. The tuples of polynomials and effective multiplicities. Let us fix the complex coordinate space $\mathbb{C}_{\left(z_{1}, \ldots, z_{N}\right)}^{N}, N \geq 1$, which we consider as embedded in the projective space $\mathbb{P}_{\left(x_{0}: x_{1}: \ldots . x_{N}\right)}^{N}$ as a standard affine chart $\left\{x_{0} \neq 0\right\}$, т.e. $z_{i}=x_{i} / x_{0}$. By the symbol $\mathcal{P}_{d, N}$ we denote the space of homogeneous polynomials of degree $d \geq 1$ in the variables $z_{*}$. For $e \leq d$ set

$$
\mathcal{P}_{[e, d], N}=\stackrel{\oplus}{i=e}_{i, N} \mathcal{P}_{i,}
$$

for instance, $\mathcal{P}_{[1, d], N}$ is the space of polynomials of degree $\leq d$ without the constant term. On each of these spaces we have an action of the matrix group $G L_{N}(\mathbb{C})$ of linear changes of coordinates. Let

$$
\mathcal{P}_{N}^{\left(n_{1}, n_{2}\right)}=\mathcal{P}_{[1,2], N}^{n_{1}} \times \mathcal{P}_{[1,3], N}^{n_{2}}=\prod_{i=1}^{n_{1}} \mathcal{P}_{[1,2], N} \times \prod_{i=1}^{n_{2}} \mathcal{P}_{[1,3], N}
$$

be the space of tuples $\left(f_{1}, \ldots, f_{n_{1}}, f_{n_{1}+1}, \ldots, f_{n_{1}+n_{2}}\right)$ of polynomials, where the first $n_{1}$ polynomialsare of degree $\leq 2$ and the subsequent $n_{2}$ polynomials are of degree at most 3 . All polynomials vanish at the point $o=(0, \ldots, 0)$. We assume that the inequality $n_{1}+n_{2}=N$ holds. 
For an effective cycle $R$ of pure codimension $l$ on $\mathbb{P}^{N}$ and a tuple of polynomials $\left(f_{1}, \ldots, f_{N}\right) \in \mathcal{P}_{N}^{\left(n_{1}, n_{2}\right)}$ define the effective multiplicity

$$
\mu_{R}\left(f_{1}, \ldots, f_{N}\right) \in \mathbb{Z}_{+} \cup\{\infty\}
$$

in the following way. If $o \notin \operatorname{Supp} R$, then we set $\mu_{R}\left(f_{*}\right)=0$. If the closed set

$$
Z_{R}\left(f_{1}, \ldots, f_{N}\right)=\left\{f_{1}=\ldots=f_{N}=0\right\} \cap R
$$

is of positive dimension at the point $o$, then we set $\mu_{R}\left(f_{*}\right)=\infty$. If none of these two cases takes place, then $\mu_{R}\left(f_{*}\right)$ is a positive integer, to define which we need some additional constructions. Let

$$
\Sigma_{1}\left(f_{*}\right)=<f_{1}, \ldots, f_{n_{1}}>\subset \mathcal{P}_{[1,2], N}
$$

be the linear span of the quadratic polynomials. Let

$$
\Sigma_{12}\left(f_{*}\right)=<f_{1} \mathcal{P}_{[0,1], N}, \ldots, f_{n_{1}} \mathcal{P}_{[0,1], N}>\subset \mathcal{P}_{[1,3], N}
$$

be the linear space of all linear combinations of the polynomials $f_{1}, \ldots, f_{n_{1}}$ with polynomials of degree at most 1 in $z_{*}$ as coefficients. In particular, we have the following inclusion: $\Sigma_{1} \subset \Sigma_{12}$. Let

$$
\Sigma_{22}\left(f_{*}\right)=<f_{n_{1}+1}, \ldots, f_{n_{1}+n_{2}}>\subset \mathcal{P}_{[1,3], N}
$$

be the linear span of the cubic polynomials and

$$
\Sigma_{2}\left(f_{*}\right)=\Sigma_{12}\left(f_{*}\right)+\Sigma_{22}\left(f_{*}\right) \subset \mathcal{P}_{[1,3], N}
$$

the sum of these two subspaces. Define the polynomial span

$$
\left[f_{1}, \ldots, f_{N}\right] \subset \mathcal{P}_{N}^{\left(n_{1}, n_{2}\right)}
$$

of the tuple $\left(f_{*}\right)$ as the set of all such tuples $\left(f_{1}^{\sharp}, \ldots, f_{N}^{\sharp}\right)$ that $f_{1}^{\sharp}, \ldots, f_{n_{1}}^{\sharp} \in \Sigma_{1}\left(f_{*}\right)$ and $f_{n_{1}+1}^{\sharp}, \ldots, f_{n_{1}+n_{2}}^{\sharp} \in \Sigma_{2}\left(f_{*}\right)$. Obviously, $\left[f_{*}\right]$ is a closed irreducible subset of the space $\mathcal{P}_{N}^{\left(n_{1}, n_{2}\right)}$.

Now for an irreducible subvariety $R \subset \mathbb{P}^{N}$ of codimension $l, R \ni o$, set

$$
\mu_{R}\left(f_{1}, \ldots, f_{N}\right)=\operatorname{dim} \mathcal{O}_{o, R} /\left(f_{l+1}^{\sharp}, \ldots, f_{N}^{\sharp}\right)
$$

where $\left(f_{1}^{\sharp}, \ldots, f_{N}^{\sharp}\right) \in\left[f_{1}, \ldots, f_{N}\right]$ is a generic tuple.For an arbitrary effective cycle $R=\Sigma_{j \in J} r_{j} R_{j}$ of pure codimension $l$ we define $\mu_{R}\left(f_{*}\right)$ by linearity, setting

$$
\mu_{R}\left(f_{*}\right)=\sum_{j \in J} r_{j} \mu_{R_{j}}\left(f_{*}\right) .
$$

The definition given above is equivalent to the following one which works for any effective cycle of codimension $l$ :

$$
\mu_{R}\left(f_{*}\right)=\operatorname{mult}_{o}\left(\left\{f_{l+1}^{\sharp}=0\right\} \circ \ldots \circ\left\{f_{N}^{\sharp}=0\right\} \circ R\right),
$$


in the brackets it is the effective zero-dimensional cycle of the scheme-theoretic intersection of the hypersurfaces $\left\{f_{i}^{\sharp}=0\right\}$, where $i=l+1, \ldots, N$, and the cycle $R$ in a neighborhood of the point $o$.

Remark 2.1. To define the effective multiplicity, one needs to form the polynomial span $\left[f_{*}\right]$, as certain components of the cycle $R$ and the projectivized tangent cone to $R$ at the point $o$ can be entirely contained in the divisors $\left\{f_{i}=0\right\}$. For that reason the numbers of the form

$$
\operatorname{dim} \mathcal{O}_{o, R} /\left(f_{i_{1}}, \ldots, f_{i_{N-l}}\right)
$$

can turn out to be strictly higher than the effective multiplicity, correctly defined above, even if $R$ is an irreducible subvariety, not contained in the hypersurfaces $\left\{f_{i}=0\right\}$.

2.2. The Chow varieties and the local effective multiplicities. By the symbol $\mathbb{H}_{l, N}(d)$ we denote the Chow variety, parametrizing effective cycles of pure codimension $l$ and degree $d$ on $\mathbb{P}^{N}$. Consider the sets

$$
\mathcal{X}_{l, N}(m, d) \subset \mathcal{P}_{[1,2], N}^{n_{1}} \times \mathcal{P}_{[1,3], N}^{n_{2}} \times \mathbb{H}_{l, N}(d),
$$

consisting of such tuples $\left(\left(f_{1}, \ldots, f_{N}\right), R\right)$, that

$$
\mu_{R}\left(f_{1}, \ldots, f_{N}\right) \geq m \in \mathbb{Z}_{+} \cup\{\infty\} .
$$

It is easy to see that $\mathcal{X}_{l, N}(m, d)$ is a closed algebraic set. Denoting by the symbol $\pi_{\mathcal{P}}$ the projection

$$
\left(\left(f_{1}, \ldots, f_{N}\right), R\right) \mapsto\left(f_{1}, \ldots, f_{N}\right),
$$

and taking into account that the Chow varieties are projective, we get that

$$
X_{l, N}(m, d)=\pi_{\mathcal{P}}\left(\mathcal{X}_{l, N}(m, d)\right) \subset \mathcal{P}_{[1,2], N}^{n_{1}} \times \mathcal{P}_{[1,3], N}^{n_{2}}
$$

is a closed algebraic set. Explicitly, it consists of such tuples $\left(f_{1}, \ldots, f_{N}\right)$, for which there exists an effective cycle $R \in \mathbb{H}_{l, N}(d)$, satisfying the inequality $\mu_{R}\left(f_{1}, \ldots, f_{N}\right) \geq$ $m$.

Let $B \subset \mathcal{P}_{[1,2], N}^{n_{1}} \times \mathcal{P}_{[1,3], N}^{n_{2}}$ be an irreducible subvariety. We define the local effective multiplicity, setting

$$
\mu_{l, N}^{\text {local }}(B, d)=\mu_{l, N}(B, d)=\max _{m \in \mathbb{Z}_{+} \cup\{\infty\}}\left\{m \mid B \subset X_{l, N}(m, d)\right\} .
$$

The explicit meaning of this definition is as follows: $\mu_{l, N}(B, d)=m$, if for a generic tuple $\left(f_{*}\right) \in B$ and any effective cycle $R \in \mathbb{H}_{l, N}(d)$ the inequality

$$
\mu_{R}\left(f_{1}, \ldots, f_{N}\right) \leq m
$$

holds and for at least one cycle $R \in \mathbb{H}_{l, N}(d)$ this inequality turns into the equality. It is clear that if $\mu_{l, N}(B, d)=\infty$, then for a generic (and thus for any) tuple $\left(f_{1}, \ldots, f_{N}\right)$ 
the set of its zeros $Z\left(f_{1}, \ldots, f_{N}\right)$ has a component of positive dimension, passing through the point $o$. The converse is also true: if there is such a component, for $R$ one can take a subvariety such that $\operatorname{dim}\left(R \cap Z\left(f_{*}\right)\right) \geq 1$.

Proposition 2.1. For any $d \geq 1$ the equality

$$
\operatorname{codim} X_{l, N}(\infty, d)=n_{1}+2 n_{2}+1 .
$$

holds.

Proof is given in $\S 5$.

Since in $\S \S 2-4$ only local multiplicities are considered, to simplify the notations we omit the indication that they are local: we write $\mu$ instead of $\mu^{\text {local }}$ or $\mu_{\text {local }}$; in $\S 5$ we will define the global multiplicities $\mu_{\text {total }}$ and for the local multiplicities we will use the notation $\mu^{\text {local }}$ or $\mu_{\text {local }}$.

Finally, set $\mu_{l, N}(a, d)=m$ for $a \in \mathbb{Z}_{+}$, if there is an irreducible subvariety $B \subset \mathcal{P}_{N}^{\left(n_{1}, n_{2}\right)}$ of codimension at most $a$, such that the equality $\mu_{l, N}(B, d)=m$ holds and the inequality

$$
\operatorname{codim} X_{l, N}(m+1, d) \geq a+1
$$

is satisfied.

Remark 2.2. By the definitions given above, if the inequality $\mu_{l, N}(a, d) \leq m$ is satisfied, it means that $\operatorname{codim} X_{l, N}(m+1, d) \geq a+1$. Explicitly: the set of such tuples $\left(f_{*}\right) \in \mathcal{P}_{N}^{\left(n_{1}, n_{2}\right)}$, that there exists an effective cycle $R$ of codimension $l$ and degree $d$, satisfying the inequality $\mu_{R}\left(f_{1}, \ldots, f_{N}\right) \geq m+1$, is of codimension at least $a+1$ in the space $\mathcal{P}_{N}^{\left(n_{1}, n_{2}\right)}$.

Obviously, by Proposition 2.1 for any $a \geq n_{1}+2 n_{2}+1$ we have $\mu_{l, N}(a, d)=\infty$ and for $a \leq n_{1}+2 n_{2}$ we have $\mu_{l, N}(a, d)<\infty$. Starting from this moment we assume that $a \leq n_{1}+2 n_{2}$. (In the sequel, we will be interested mainly in the case $a=n_{1}+n_{2}=N$.)

By construction, the sets $X_{l, N}(m, d)$ are invariant under the linear changes of coordinates (the group $G L_{N}(\mathbb{C})$ ). Besides, these sets are invariant under the action of another group $G\left(n_{1}, n_{2}\right)$ on the space $\mathcal{P}_{N}^{\left(n_{1}, n_{2}\right)}$, which we will now define. The group $G\left(n_{1}, n_{2}\right)$ is an extension

$$
\begin{gathered}
1 \rightarrow \underset{[0,1], N}{\times\left(n_{1} n_{2}\right)} \rightarrow G\left(n_{1}, n_{2}\right) \rightarrow G L_{n_{1}}(\mathbb{C}) \times G L_{n_{2}}(\mathbb{C}) \rightarrow 1 . \\
\| \\
\mathbb{C}^{n_{1} n_{2}(N+1)}
\end{gathered}
$$

More precisely, to an element $g \in G\left(n_{1}, n_{2}\right)$ corresponds a triple of matrices $\left(A_{11}, A_{12}, A_{22}\right)$, where

$$
A_{11} \in G L_{n_{1}}(\mathbb{C}), \quad A_{22} \in G L_{n_{2}}(\mathbb{C}), \quad A_{12} \in \operatorname{Mat}_{\left(n_{1}, n_{2}\right)}\left(\mathcal{P}_{[0,1], N}\right) .
$$

Setting $A_{11}=\left\|\alpha_{i j}\right\|_{1 \leq i, j \leq n_{1}}, A_{22}=\left\|\beta_{i j}\right\|_{1 \leq i, j \leq n_{2}}$ and

$$
A_{12}=\left\|\gamma_{i j}\left(z_{1}, \ldots, z_{N}\right)\right\|_{1 \leq i \leq n_{1}, 1 \leq j \leq n_{2}},
$$


we define the action

$$
g: f=\left(f_{1}, \ldots, f_{N}\right) \mapsto f^{g}=\left(f_{1}^{g}, \ldots, f_{N}^{g}\right) \in \mathcal{P}_{N}^{\left(n_{1}, n_{2}\right)}
$$

by the formulas

$$
\begin{gathered}
f_{i}^{g}=\sum_{j=1}^{n_{1}} \alpha_{i j} f_{j}, \quad i=1, \ldots, n_{1}, \\
f_{n_{1}+i}^{g}=\sum_{j=1}^{n_{2}} \beta_{i j} f_{n_{1}+j}+\sum_{i=1}^{n_{1}} \gamma_{i j}\left(z_{*}\right) f_{i}, \quad j=1, \ldots, n_{2} .
\end{gathered}
$$

The closed subset $B \subset \mathcal{P}_{N}^{\left(n_{1}, n_{2}\right)}$ is said to be bi-invariant, if it is invariant under the action of both groups, $G L_{N}(\mathbb{C})$ (changes of coordinates) and $G\left(n_{1}, n_{2}\right)$. In particular, the sets $X_{l, N}(m, d)$ are bi-invariant (the multiplicities $\mu_{R}\left(f_{*}\right)$ are obviously invariant under the action of both groups). Note that the group $G\left(n_{1}, n_{2}\right)$ contains the subgroup $\bar{G}\left(n_{1}, n_{2}\right) \subset G L_{N}(\mathbb{C})$, corresponding to the tuples of matrices $\left(A_{11}, A_{12}, A_{22}\right) \mathrm{c}$

$$
A_{12} \in \operatorname{Mat}_{\left(n_{1}, n_{2}\right)}(\mathbb{C}) \subset \operatorname{Mat}_{\left(n_{1}, n_{2}\right)}\left(\mathcal{P}_{[0,1], N}\right) .
$$

2.3. Reducing to the standard form. For an irreducible subvariety $B \subset$ $\mathcal{P}_{N}^{\left(n_{1}, n_{2}\right)}$ we define its type

$$
\tau(B)=\left(\left(a_{1}, b_{1}\right),\left(a_{2}, b_{2}\right)\right) \in \mathbb{Z}_{+}^{\times 4}
$$

setting: $a_{1}=\operatorname{codim}\left(\operatorname{pr}_{1}(B) \subset \mathcal{P}_{[1,2], N}^{n_{1}}\right)$, where $\operatorname{pr}_{1}: \mathcal{P}_{N}^{\left(n_{1}, n_{2}\right)} \rightarrow \mathcal{P}_{[1,2], N}^{n_{1}}$ is the projection onto the first $n_{1}$ direct factors; $a_{2}=a-a_{1}$, where $a=\operatorname{codim}\left(B \subset \mathcal{P}_{N}^{\left(n_{1}, n_{2}\right)}\right)$;

$$
b_{1}=n_{1}-\operatorname{rk}\left(d f_{1}(o), \ldots, d f_{n_{1}}(o)\right)
$$

for a tuple of general position $\left(f_{1}, \ldots, f_{n_{1}}\right) \in \operatorname{pr}_{1}(B) ; b_{2}=b-b_{1}$, where

$$
b=\varepsilon(B)=N-\operatorname{rk}\left(d f_{1}(o), \ldots, d f_{N}(o)\right)
$$

for a tuple of general position $\left(f_{1}, \ldots, f_{N}\right) \in B$. If the subvariety $B$ is $G\left(n_{1}, n_{2}\right)$ (or at least $\left.\bar{G}\left(n_{1}, n_{2}\right)\right)$-invariant, then in a generic tuple of $N$ polynomials $\left(f_{1}, \ldots, f_{N}\right) \in B$ the linear forms

$$
d f_{1}(o), \ldots, d f_{n_{1}-b_{1}}(o), d f_{n_{1}+1}(o) \ldots, d f_{N-b_{2}}(o)
$$

are linearly independent, the forms

$$
d f_{n_{1}-b_{1}+1}(o), \ldots, d f_{n_{1}}(o)
$$

are linear combinations of $d f_{1}(o), \ldots, d f_{n_{1}-b_{1}}(o)$, and the forms

$$
d f_{N-b_{2}+1}(o), \ldots, d f_{N}(o)
$$


are linear combinations of the forms (10). Therefore, for an arbitrary $G\left(n_{1}, n_{2}\right)$ (or $\left.\bar{G}\left(n_{1}, n_{2}\right)\right)$-invariant irreducible subvariety $B$ there exists a non-empty Zarisky open subset $B^{\circ} \subset B$, on which the following map of reducing to the standard form is well defined:

$$
\rho: B^{o} \rightarrow \mathcal{P}_{[1,2], N}^{n_{1}-b_{1}} \times \mathcal{P}_{2, N}^{b_{1}} \times \mathcal{P}_{[1,3], N}^{n_{2}-b_{2}} \times \mathcal{P}_{[2,3], N}^{b_{2}},
$$

so that this map transforms a generic tuple $\left(f_{1}, \ldots, f_{N}\right)$ into the tuple of polynomials

$$
\left(f_{1}, \ldots, f_{n_{1}-b_{1}}, f_{n_{1}-b_{1}+1}^{+}, \ldots, f_{n_{1}}^{+}, f_{n_{1}+1}, \ldots, f_{N-b_{2}}, f_{N-b_{2}+1}^{+}, \ldots, f_{N}^{+}\right)
$$

where $f_{i}^{+}$for $i \in\left\{n_{1}-b_{1}+1, \ldots, n_{1}\right\}$ are obtained by subtracting from $f_{i}$ the uniquely determined linear combination of polynomials $f_{1}, \ldots, f_{n_{1}-b_{1}}$, as a result of which $d f_{i}^{+}(o)=0$, and $f_{i}^{+}$for $i \in\left\{N-b_{2}+1, \ldots, N\right\}$ are obtained by subtracting from $f_{i}$ the uniquely determined linear combination of polynomials $f_{j}, j \in\left\{1, \ldots, n_{1}-\right.$ $\left.b_{1}, n_{1}+1, \ldots, N-b_{2}\right\}$, so that $d f_{i}^{+}(o)=0$. We denote the closure of the image $\rho\left(B^{o}\right)$ by the symbol $\bar{B}$. If the subvariety $B$ is invariant with respect to the action of $\bar{G}\left(n_{1}, n_{2}\right)$, then the coefficients of the above mentioned linear combinations can be arbitrary and for that reason

$$
\begin{aligned}
\operatorname{dim} B & =\operatorname{dim} \bar{B}+b_{1}\left(n_{1}-b_{1}\right)+b_{2}(N-b)= \\
& =\operatorname{dim} \bar{B}+b\left(n_{1}-b_{1}\right)+b_{2}\left(n_{2}-b_{2}\right) .
\end{aligned}
$$

On the other hand, the direct product in (11) is of codimension $b N$ in the space $\mathcal{P}_{N}^{\left(n_{1}, n_{2}\right)}$. Therefore, the equality

$$
\operatorname{codim} \bar{B}=\operatorname{codim} B-b_{1}\left(n_{2}+b_{1}\right)-b_{2} b
$$

holds. In the last formula the codimension of each of the subvarieties is meant with respect to the relevant ambient space: for $B$ it is $\mathcal{P}_{N}^{\left(n_{1}, n_{2}\right)}$, for $\bar{B}$ it is the direct product in (11). Starting from this moment, unless otherwise specified, the codimension is always meant with respect to the natural ambient space. Sometimes, for the convenience of the reader, we remind, with respect to what ambient space the codimension is meant.

Obviously, every fibre of the map $\rho: B^{o} \rightarrow \rho\left(B^{o}\right)$ is $\mathbb{C}^{\left(b_{1}\left(n_{1}-b_{1}\right)+b_{2}(N-b)\right)}$.

By construction,

$$
\mu_{l, N}(a, d)=\max _{B} \mu_{l, N}(B, d),
$$

where the maximum is taken over all bi-invariant irreducible subvarieties $B \subset$ $\mathcal{P}_{N}^{\left(n_{1}, n_{2}\right)}$ of codimension at most $a$. Our method of estimating the numbers $\mu_{l, N}(B, d)$ (and thus the numbers $\mu_{l, N}(a, d)$ ) is based on controlling the type $\tau(B)$ of these subvarieties. It is easy to see that the conditions

$$
\operatorname{rk}\left(d f_{1}(o), \ldots, d f_{n_{1}}(o)\right) \leq n_{1}-b_{1}
$$

and

$$
\operatorname{rk}\left(d f_{1}(o), \ldots, d f_{N}(o)\right) \leq N-b
$$


define in $\mathcal{P}_{N}^{\left(n_{1}, n_{2}\right)}$ a bi-invariant irreducible subvariety of the type $\left(\left(a_{1}^{*}, b_{1}\right),\left(a_{2}^{*}, b_{2}\right)\right)$, where

$$
a_{1}^{*}=b_{1}\left(N+b_{1}-n_{1}\right) \quad \text { and } \quad a_{2}^{*}=b^{2}-a_{1}^{*} .
$$

Therefore, a bi-invariant irreducible subvariety $B$ of the type $\tau(B)=\left(\left(a_{1}, b_{1}\right),\left(a_{2}, b_{2}\right)\right)$ can exist only if the inequalities

$$
a_{1} \geq b_{1}\left(N+b_{1}-n_{1}\right) \text { and } \quad a=a_{1}+a_{2} \geq b^{2}
$$

hold.

Proposition 2.2. Assume that a (bi-invariant) subvariety $B$ is of the type $\tau(B)=\left(\left(a_{1}, 0\right),\left(a_{2}, 0\right)\right)$, that is, $b=0$. Then

$$
\mu_{l, N}(B, d)=d
$$

for any $d \geq 1$.

Proof. Let $R \ni o$ be an irreducible subvariety in $\mathbb{P}^{N},\left(f_{1}, \ldots, f_{N}\right) \in B$ a generic tuple of polynomials. By assumption,

$$
\operatorname{rk}\left(d f_{1}(o), \ldots, d f_{N}(o)\right)=N
$$

Set $\Pi=\left\{d f_{1}(o)=\ldots=d f_{n_{1}}(o)=0\right\} \subset \mathbb{C}^{N}$, this is a linear subspace of codimension $n_{1}$. Let

$$
\left(f_{1}^{\sharp}, \ldots, f_{N}^{\sharp}\right) \in\left[f_{1}, \ldots, f_{N}\right] \subset \mathcal{P}_{N}^{\left(n_{1}, n_{2}\right)}
$$

be a generic element of the polynomial span of the tuple $\left(f_{*}\right)$. By construction, the forms $d f_{1}^{\sharp}(o), \ldots, d f_{n_{1}}^{\sharp}(o)$ are linear forms of general position, vanishing on the space $\Pi$, and $d f_{n_{1}+1}^{\sharp}(o), \ldots, d f_{N}^{\sharp}(o)$ are arbitrary linear forms of general position.

If $n_{1} \leq l$, then the inequality

$$
\mu_{R}\left(f_{*}\right)=\operatorname{dim} \mathcal{O}_{o, R} /\left(f_{l+1}^{\sharp}, \ldots, f_{N}^{\sharp}\right)=\operatorname{mult}_{o} R
$$

holds, since the differentials $d f_{i}^{\sharp}(o), i=l+1, \ldots, N$, are forms of general position. If $n_{1}>l$, then for the first $n_{1}-l$ differentials $d f_{i}^{\sharp}(o)$, where $i=l+1, \ldots, n_{1}$, there is a (unique) constraint

$$
\left.d f_{i}^{\sharp}(o)\right|_{\Pi} \equiv 0 .
$$

Let $T_{o} R \subset \mathbb{C}^{N}$ be the algebraic tangent cone to $R$ at the point $o$. Each of its components has codimension $l$. Since $\operatorname{codim}\left(\Pi \subset \mathbb{C}^{N}\right)=n_{1}$, for a generic $f_{l+1}^{\sharp}$ one may assume that the linear form $d f_{l+1}^{\sharp}$ vanishes on no components of the cone $T_{o} R$. Therefore,

$$
\operatorname{mult}_{o}\left(R \circ\left\{f_{l+1}^{\sharp}=0\right\}\right)=\operatorname{mult}_{o} R .
$$

For the same reasons, for generic $f_{l+1}^{\sharp}, \ldots, f_{n_{1}}^{\sharp}$ we have the equality

$$
\operatorname{mult}_{o}\left(R \circ\left\{f_{l+1}^{\sharp}=0\right\} \circ \ldots \circ\left\{f_{l+1}^{\sharp}=0\right\}\right)=\operatorname{mult}_{o} R \text {. }
$$


Now the equality $\mu_{R}\left(f_{*}\right)=$ mult $_{o} R$ is obtained as in the case $n_{1} \leq l$. Since mult S $_{o} R \leq$ $\operatorname{deg} R$ and the equality is attained, Proposition 2 is proved. Q.E.D.

Note that if the multiplicity $\mu_{l, N}(B, d)$ is finite, then it does not exceed the number $2^{a_{1}-l} 3^{a_{2}} d$ for $a_{1} \geq l$ and the number $3^{a-l} d$ for $a_{1}<l$. However, these estimates are too weak for our purposes. Below the following key facts will be shown.

Theorem 4. The following inequalities are true:

$$
\mu_{l, N}(B, d) \leq \frac{1}{2 \pi b}\left(\frac{2 a-b(b-1)}{b^{2}} e^{2}\right)^{b} d<\frac{1}{2 \pi b}\left(\frac{2 a}{b^{2}} e^{2}\right)^{b} d .
$$

and

$$
\mu_{l, N}(a, d) \leq \frac{e^{2}}{2 \pi[\sqrt{a}]}\left(2 e^{2}\right)^{[\sqrt{a}]} d
$$

Theorem 5. For $a \geq 17$ the following estimate holds:

$$
\mu_{l, N}(a, d) \leq \frac{e^{2}}{2 \pi[\sqrt{a}]}\left(\frac{5}{3} e^{2}\right)^{[\sqrt{a}]} d
$$

Therefore, for a fixed $l$ and $a \rightarrow \infty$ the effective multiplicity has exponential growth of the order $\sqrt{a}$, and not of the order $a$, as in the aprioric estimates above.

\section{§3. Local multiplicities. II.}

\section{The inductive method of estimating}

In this section, we construct the key procedure of estimating the local multiplicity in terms of the local multiplicities of the truncated tuples of polynomials.

3.1. Splitting off a direct factor. If $b_{2} \geq 1$, then denote by the symbol $\pi_{2}$ the projection

$$
\begin{array}{rll}
\mathcal{P}_{[1,2], N}^{n_{1}-b_{1}} \times \mathcal{P}_{2, N}^{b_{1}} & \times & \mathcal{P}_{[1,3], N}^{n_{2}-b_{2}} \times \mathcal{P}_{[2,3], N}^{b_{2}} \\
& \downarrow & \\
\mathcal{P}_{[1,2], N}^{n_{1}-b_{1}} \times \mathcal{P}_{2, N}^{b_{1}} & \times & \mathcal{P}_{[1,3], N}^{n_{2}-b_{2}} \times \mathcal{P}_{[2,3], N}^{b_{2}-1}
\end{array}
$$

along the last direct factor $\mathcal{P}_{[2,3], N}$. If $b_{1} \geq 1$, then by the symbol $\pi_{1}$ denote the similar projection along the last quadratic direct factor $\mathcal{P}_{2, N}$ onto $\mathcal{P}_{[1,2], N}^{n_{1}-b_{1}} \times \mathcal{P}_{2, N}^{b_{1}-1} \times$ $\mathcal{P}_{[1,3], N}^{n_{2}} \times \mathcal{P}_{[2,3], N}^{b_{2}}$. For a closed set $\bar{B}$, constructed above by reducing to the standard form, denote by the symbol $[\bar{B}]_{N-1}^{(i)}$ the closure of the set $\pi_{i}(\bar{B})$ in the corresponding ambient space, $i=1,2$. Furthermore, let $\lambda_{2}$ and $\lambda_{1}$ denote the projections, complementary to $\pi_{2}$ and $\pi_{1}$, that is, the projections onto the last direct factor and the last quadratic direct factor, respectively. That is to say, $\pi_{2} \times \lambda_{2}$ and $\pi_{1} \times \lambda_{1}$ are the identity map of the direct product $\mathcal{P}_{[1,2], N}^{n_{1}-b_{1}} \times \mathcal{P}_{2, N}^{b_{1}} \times \mathcal{P}_{[1,3], N}^{n_{2}-b_{2}} \times \mathcal{P}_{[2,3], N}^{b_{2}}$. 
Note that since the set $B$ is bi-invariant, the closed set $\bar{B}$ is invariant with respect to the group $G L_{N}(\mathbb{C})$ of the linear changes of coordinates and to the subgroup $G\left(n_{1}, b_{1} ; n_{2}, b_{2}\right) \subset G\left(n_{1}, n_{2}\right)$, the elements of which correspond to such triples of matrices $\left(A_{11}, A_{12}, A_{22}\right)$, that $A_{11} \in G L_{n_{1}-b_{1}}(\mathbb{C})$ (and these matrices act on the polynomials $f_{1}, \ldots, f_{n_{1}-b_{1}}$, mapping $f_{n_{1}-b_{1}+1}, \ldots, f_{n_{1}}$ to themselves), $A_{22} \in G L_{n_{2}-b_{2}}(\mathbb{C})$ (these matrices act on $\left.f_{n_{1}+1}, \ldots, f_{n_{1}+n_{2}-b_{2}}\right)$ and $A_{12} \in \operatorname{Mat}_{\left(n_{1}, n_{2}\right)}\left(\mathcal{P}_{[0,1], N}\right)$, where only the polynomials $\gamma_{i j}$ c $j=1, \ldots, n_{2}-b_{2}$ have an arbitrary constant term, for $j \geq n_{2}-b_{2}+1$ these polynomials are homogeneous: $\gamma_{i j} \in \mathcal{P}_{1, N}$. This trivial remark is used below for estimating the parameters of the variety $B_{h} \subset \mathcal{P}_{N-1}^{\left(n_{1}, n_{2}-1\right)}$ without special comments.

To simplify notations, assume that $b_{2} \geq 1$, consider the projection $\pi_{2}$ and write $[\bar{B}]_{N-1}$ instead of $[\bar{B}]_{N-1}^{(2)}$. The modifications required in the case $i=1$ are obvious and we will give only the final result. In all detail we consider the case $i=2$.

For a generic tuple $\left(f_{1}, \ldots, f_{N-1}\right) \in[\bar{B}]_{N-1}$ by the symbol

$$
[\bar{B}]^{N}=[\bar{B}]^{N}\left(f_{1}, \ldots, f_{N-1}\right) \subset \mathcal{P}_{[2,3], N}
$$

we denote the fibre of the projection $\left.\pi_{2}\right|_{\bar{B}}: \bar{B} \rightarrow[\bar{B}]_{N-1}$. Obviously,

$$
\operatorname{codim} \bar{B}=\operatorname{codim}[\bar{B}]_{N-1}+\operatorname{codim}[\bar{B}]^{N}
$$

(recall: the codimension is meant with respect to the relevant ambient space, for instance, for $[\bar{B}]^{N}$ it is the fibre of the projection $\pi_{2}$, that is, $\left.\mathcal{P}_{[2,3], N}\right)$. Set $\gamma_{N}=$ $\gamma_{N}(B)=\operatorname{codim}[\bar{B}]^{N}$. Since $\operatorname{codim} B \leq a$, we obtain (see (12)) the estimate

$$
\operatorname{codim}[\bar{B}]_{N-1} \leq a-b_{1}\left(n_{2}+b_{1}\right)-b_{2} b-\gamma_{N} .
$$

From this, in particular, follows the inequality

$$
0 \leq \gamma_{N} \leq a-b_{1}\left(n_{2}+b_{1}\right)-b_{2} b
$$

Let $h\left(z_{*}\right) \in \mathcal{P}_{1, N}$ be a non-zero linear form, $H=\{h=0\} \subset \mathbb{C}^{N}$ the corresponding hyperplane. By one and the same symbol $\pi_{h}$ we will denote the projection of all spaces $\mathcal{P}_{[i, j], N}$ onto $\mathcal{P}_{[i, j], N-1}$ and the corresponding projections for direct products of these spaces, where it is meant that an isomorphism $H \cong \mathbb{C}^{N-1}$ is fixed and

$$
\pi_{h}:\left.f \mapsto f\right|_{H}
$$

is the restriction of the polynomial onto $H$.

Let $B_{h} \subset \mathcal{P}_{N-1}^{\left(n_{1}, n_{2}-1\right)}$ be the smallest bi-invariant (in the sense of the latter space, that is, invariant under the action of the groups $G L_{N-1}(\mathbb{C})$ and $G\left(n_{1}, n_{2}-1\right)$ ) closed set, containing the set $\pi_{h}\left([\bar{B}]_{N-1}\right)$. Obviously, $B_{h}$ is a bi-invariant irreducible subvariety. For a generic tuple $\left(f_{1}, \ldots, f_{N-1}\right) \in[\bar{B}]_{N-1}$ consider the following tuple of linear forms

$$
\left.d f_{1}(o)\right|_{H}, \ldots,\left.d f_{n_{1}-b_{1}}(o)\right|_{H},\left.d f_{n_{1}+1}(o)\right|_{H}, \ldots,\left.d f_{N-b_{2}}(o)\right|_{H}
$$


The rank of this tuple is either $N-b$, or $N-b-1$. More precisely, there exists an integer-valued vector

$$
\alpha=\left(\alpha_{1}, \alpha_{2}\right) \in\{(0,1),(-1,1),(0,0)\}
$$

such that for the parameters $\left(b_{1}^{h}, b_{2}^{h}\right)$ of the variety $B_{h}$ the following equalities hold:

$$
b_{1}^{h}=b_{1}-\alpha_{1}, \quad b_{2}^{h}=b_{2}-\alpha_{2} .
$$

Let $\tau\left(B_{h}\right)=\left(\left(a_{1}^{h}, b_{1}^{h}\right),\left(a_{2}^{h}, b_{2}^{h}\right)\right)$ be the type of the subvariety $B_{h}$. Furthermore, set $b^{h}=b_{1}^{h}+b_{2}^{h}$ and $a^{h}=a_{1}^{h}+a_{2}^{h}$. Obviously, $a^{h}$ is the codimension of the subvariety $B_{h}$ in the ambient space $\mathcal{P}_{N-1}^{\left(n_{1}, n_{2}-1\right)}$.

To realize the inductive procedure defined in Sec. 3.3 below, we will need, apart from $B_{h}$, another family of subvarieties in the space $\mathcal{P}_{N-1}^{\left(n_{1}, n_{2}-1\right)}$, which we will now introduce, again for the case $i=2$, with the obvious modifications in the case $i=1$. Take any polynomial $f \in \lambda_{2}(\bar{B}) \subset \mathcal{P}_{[2,3], N}$ and consider the closed set

$$
\bar{B}(f)=\lambda_{2}^{-1}(f) \cap \bar{B} \subset \mathcal{P}_{[1,2], N}^{n_{1}-b_{1}} \times \mathcal{P}_{2, N}^{b_{1}} \times \mathcal{P}_{[1,3], N}^{n_{2}-b_{2}} \times \mathcal{P}_{[2,3], N}^{b_{2}-1}
$$

We will consider the product in the right hand side of the last inclusion as the natural ambient space for $\bar{B}(f)$; in particular, the codimension of $\bar{B}(f)$ is meant with respect to that space. By construction, $\bar{B}(f) \neq \emptyset$ and

$$
\operatorname{codim} \bar{B} \geq \operatorname{codim} \bar{B}(f)+\operatorname{codim} \lambda_{2}(\bar{B}),
$$

so that we obtain the estimate

$$
\operatorname{codim} \bar{B}(f) \leq a-b_{1}\left(n_{2}+b_{1}\right)-b_{2} b-\gamma(f)
$$

for some $\gamma(f) \in \mathbb{Z}_{+}, \gamma(f) \geq \operatorname{codim} \lambda_{2}(\bar{B})$. Once again, take a non-zero linear form $h\left(z_{*}\right) \in \mathcal{P}_{1, N}$ with $H=\{h=0\}$ the corresponding hyperplane, fix an isomorphism $H \cong \mathbb{C}^{N-1}$ and let $\pi_{h}$ mean the same as above.

Let $B_{h}(f) \subset \mathcal{P}_{N-1}^{\left(n_{1}, n_{2}-1\right)}$ be the smallest bi-invariant (in the sense of the latter space, that is, invariant under the action of the groups $G L_{N-1}(\mathbb{C})$ and $G\left(n_{1}, n_{2}-1\right)$ ) closed set, containing the set $\pi_{h}(\bar{B}(f))$. Obviously, $B_{h}(f) \subset B_{h}$ (since $\bar{B}(f) \subset$ $\left.[\bar{B}]_{N-1}\right)$ is a bi-invariant closed subset, which we without loss of generality can assume to be an irreducible subvariety. We will put off a discussion of the invariants of the subvarieties $B_{h}(f)$ until Sec. 3.3, because we will use the polynomials $f$ of a special form there. Now let us study the subvariety $B_{h}$ in more detail.

3.2. Estimating the codimension of the subvariety $B^{h}$. How the type of the subvariety $B$ changes when we restrict onto a hyperplane, is shown in the following

Proposition 3.1. (i) In the case $\alpha=(0,1)$ the estimates

$$
a_{1}^{h} \leq a_{1}-b_{1}, \quad a^{h} \leq a-(2 b-1)-\gamma_{N}
$$

hold. 
(ii) In the case $\alpha=(-1,1)$ the estimates

$$
a_{1}^{h} \leq a_{1}, \quad a^{h} \leq a-b-\gamma_{N}
$$

hold.

(iii) In the case $\alpha=(0,0)$ the estimates

$$
a_{1}^{h} \leq a_{1}-b_{1}, \quad a^{h} \leq a-b_{1}-b-\gamma_{N}
$$

hold.

Proof. We will study in full detail the cases (i) and (ii), in the case (iii) we only give the computations. So assume that $\alpha=(0,1)$. This means that the forms $d f_{i}(o)$, $i \in\left\{1, \ldots, n_{1}-b_{1}, n_{1}+1, \ldots, N-b_{2}\right\}$, remain linearly independent when restricted onto $H$. We get the estimate

$$
\operatorname{codim} \pi_{h}\left([\bar{B}]_{N-1}\right) \leq \operatorname{codim}[\bar{B}]_{N-1} \leq a-b_{1}\left(n_{2}+b_{1}\right)-b_{2} b-\gamma_{N} .
$$

The codimension in the left hand side is the codimension with respect to the ambient space

$$
\mathcal{P}_{[1,2], N-1}^{n_{1}-b_{1}} \times \mathcal{P}_{2, N-1}^{b_{1}} \times \mathcal{P}_{[1,3], N-1}^{n_{2}-b_{2}} \times \mathcal{P}_{[2,3], N-1}^{b_{2}-1},
$$

and moreover, for a generic tuple $\left(g_{1}, \ldots, g_{N-1}\right) \in \pi_{h}\left([\bar{B}]_{N-1}\right)$ the linear forms $d g_{i}(o)$, $i \in\left\{1, \ldots, n_{1}-b_{1}, n_{1}+1, \ldots, N-b_{2}\right\}$, are linearly independent. Now let us apply the procedure, inverse to the procedure of reducing to the standard form: the set $B_{h}$, due to its bi-invariance, certainly contains all the tuples of the form $\left(g_{1}^{+}, \ldots, g_{N-1}^{+}\right)$, where $g_{i}^{+}=g_{i}$ for $1 \leq i \leq n_{1}=b_{1}$,

$$
g_{i}^{+}=g_{i}+\sum_{j=1}^{n_{1}-b_{1}} \lambda_{i j} g_{j}
$$

for $i=n_{1}-b_{1}+1, \ldots, n_{1}$, where the coefficients $\lambda_{i j}$ are arbitrary. Furthermore, $g_{i}^{+}=g_{i}$ for $n_{1}+1 \leq i \leq N-b_{2}$ and

$$
g_{i}^{+}=g_{i}+\sum_{j=1}^{n_{1}-b_{1}} \lambda_{i j} g_{j}+\sum_{j=n_{1}+1}^{N-b_{2}} \lambda_{i j} g_{j}
$$

for $i=N-b_{2}+1, \ldots, N-1$, the coefficients $\lambda_{i j}$ are arbitrary. For that reason, the inequality

$$
\operatorname{dim} B_{h} \geq \operatorname{dim} \pi_{h}\left([\bar{B}]_{N-1}\right)+b_{1}\left(n_{1}-b_{1}\right)+\left(b_{2}-1\right)(N-b)
$$

holds. On the other hand, the dimension of the space $\mathcal{P}_{N-1}^{\left(n_{1}, n_{2}-1\right)}$ is higher than the dimension of the space (15) by $(b-1)(N-1)$. As a result we get:

$$
\begin{aligned}
a^{h} \leq & a-b_{1}\left(n_{2}+b_{1}\right)-b_{2} b-\gamma_{N}- \\
& -b_{1}\left(n_{1}-b_{1}\right)-\left(b_{2}-1\right)(N-b)+(b-1)(N-1)= \\
& =a-(2 b-1)-\gamma_{N},
\end{aligned}
$$


as it was claimed. The estimate for $a_{1}^{h}$ is obtained by similar (but simpler) arguments. The procedure of reducing to the standard form is well defined on the quadratic components, so that for the projection $\operatorname{pr}_{1}\left([\bar{B}]_{N-1}\right)$ we get the estimate

$$
\operatorname{codim} \operatorname{pr}_{1}\left([\bar{B}]_{N-1}\right) \leq a_{1}-b_{1}\left(n_{2}+b_{1}\right) .
$$

Note that for this closed set the ambient space is the direct product

$$
\mathcal{P}_{[1,2], N}^{n_{1}-b_{1}} \times \mathcal{P}_{2, N}^{b_{1}}
$$

Now, restricting onto the hyperplane $H$ and applying, as we did it above, the procedure, inverse to the procedure of reducing to the standard form, we obtain the required estimate:

$$
a^{h} \leq a_{1}-b_{1}\left(n_{2}+b_{1}\right)-b_{1}\left(n_{1}-b_{1}\right)+b_{1}(N-1)=a_{1}-b_{1},
$$

as required.

Let us consider the case (ii). Here for a generic tuple $\left(f_{1}, \ldots, f_{N-1}\right) \in[\bar{B}]_{N-1}$ the rank of the system of linear forms $\left(d f_{*}(o)\right)$ drops by one when we restrict onto $H$, and already the forms

$$
\left.d f_{1}(o)\right|_{H}, \ldots,\left.d f_{n_{1}-b_{1}}(o)\right|_{H}
$$

are linearly independent. By the $G\left(n_{1}, b_{1} ; n_{2}, b_{2}\right)$-invariance of the set $[\bar{B}]_{N-1}$ we may assume that for a generic tuple $\left(g_{1}, \ldots, g_{N-1}\right) \in \pi_{h}\left([\bar{B}]_{N-1}\right)$ the first $n_{1}-b_{1}-1$ forms $d g_{i}(o), i=1, \ldots, n_{1}-b_{1}-1$, are linearly independent, and $d g_{n_{1}-b_{1}}(o)$ is their linear combination, and moreover, for any $\lambda_{1}, \ldots, \lambda_{n_{1}-b_{1}-1}$

$$
\left(g_{1}, \ldots, g_{n_{1}-b_{1}-1}, g_{n_{1}-b_{1}}+\sum_{i=1}^{n_{1}-b_{1}-1} \lambda_{i} g_{i}, g_{n_{1}-b_{1}+1}, \ldots, g_{N-1}\right) \in \pi_{h}\left([\bar{B}]_{N-1}\right)
$$

Therefore, to the Zariski open subset

$$
\mathrm{rk}<d g_{1}(o), \ldots, d g_{n_{1}-b_{1}-1}(o)>=n_{1}-b_{1}-1
$$

one can apply the map of reducing to the standard form in the component $g_{n_{1}-b_{1}}$ and, taking the closure, obtain the irreducible subset

$$
\overline{\pi_{h}\left([\bar{B}]_{N-1}\right)} \subset \mathcal{P}_{[1,2], N-1}^{n_{1}-b_{1}-1} \times \mathcal{P}_{2, N-1}^{b_{1}+1} \times \mathcal{P}_{[1,3], N-1}^{n_{2}-b_{2}} \times \mathcal{P}_{[2,3], N-1}^{b_{2}-1}
$$

of codimension

$$
\operatorname{codim} \pi_{h}\left([\bar{B}]_{N-1}\right)-N+n_{1}-b_{1} .
$$

After that, one can apply the procedure, inverse to that of reducing to the standard form, similar to how it was done in the case (i), and obtain for the codimension of the set $B_{h}$ the estimate

$$
\operatorname{codim} B_{h} \leq a-b_{1}\left(n_{2}+b_{1}\right)-b_{2} b-\gamma_{N}-N+n_{1}-b_{1}+b(N-1)-
$$




$$
-\left(b_{1}+1\right)\left(n_{1}-b_{1}-1\right)-\left(b_{2}-1\right)(N-b-1)=a-b-\gamma_{N},
$$

as we claimed. For $a_{1}^{h}$ the arguments are similar, but simpler, since we take into account only the quadratic polynomials. Again one have to reduce the generic tuple $\left(g_{1}, \ldots, g_{n_{1}}\right)$ to the standard form in the component $g_{n_{1}-b_{1}}$ and after that apply to the resulting set the procedure, inverse to that of reducing to the standard form. As a result, we obtain the inequality

$$
\begin{aligned}
a_{1}^{h} \leq & a_{1}-b_{1}\left(n_{2}+b_{1}\right)-N+n_{1}-b_{1}- \\
& -\left(b_{1}+1\right)\left(n_{1}-b_{1}-1\right)+\left(b_{1}+1\right)(N-1)=a_{1},
\end{aligned}
$$

as it was claimed (recall that $N=n_{1}+n_{2}$ ).

Finally, let us consider the case (iii). As in the previous case, one has to complete reducing the set $\pi_{h}\left([\bar{B}]_{N-1}\right)$ to the standard form, now in the component $g_{N-b_{2}}$, since for a generic tuple $\left(g_{1}, \ldots, g_{N-1}\right)$ the linear forms

$$
d g_{1}(o), \ldots, d g_{n_{1}-b_{1}}(o), d g_{n_{1}+1}(o), \ldots, d g_{N-b_{2}-1}(o)
$$

are linearly independent, and $d g_{N-b_{2}}(o)$ is their linear combination. After that we apply the procedure, inverse to that of reducing to the standard form. We obtain the inequality:

$$
\begin{aligned}
a^{h} \leq & a-b_{1}\left(n_{2}+b_{1}\right)-b_{2} b-\gamma_{N}- \\
& -(N-1)+(N-b-1)- \\
& -b_{1}\left(n_{1}-b_{1}\right)-b_{2}(N-b-1)+b(N-1),
\end{aligned}
$$

where the second line corresponds to the reduction to the standard form and the third to the inverse procedure. Simplifying, we obtain the estimate

$$
a^{h} \leq a-b_{1}-b-\gamma_{N}
$$

as it was claimed. For the codimension $a_{1}^{h}$ in the quadratic components this case is very easy, as there is no need to reduce to the standard form. Applying the procedure, inverse to the procedure of reducing to the standard form, we obtain the inequality:

$$
a_{1}^{h} \leq a_{1}-b_{1}\left(n_{2}+b_{1}\right)-b_{1}\left(n_{1}-b_{1}\right)+b_{1}(N-1)=a_{1}-b_{1},
$$

as it was claimed. Q.E.D. for Proposition 3.1.

Now let us consider the problem of estimating the codimension when a quadratic factor is being split off. Here $\left(b_{1}^{h}, b_{2}^{h}\right)=\left(b_{1}, b_{2}\right)-\left(\alpha_{1}, \alpha_{2}\right)$, where $\alpha=\left(\alpha_{1}, \alpha_{2}\right)$ can take the values $(1,0),(1,-1)$ and $(0,0)$. The inequalities for $a^{h}$ and $a_{1}^{h}$ are obtained by word for word the same arguments as when a cubic factor was split off. We give the final result.

Proposition 3.2. (i) In the case $\alpha=(1,0)$ the estimates

$$
a_{1}^{h} \leq a_{1}-n_{2}-2 b_{1}+1-\gamma_{N}, \quad a^{h} \leq a-n_{2}-2 b_{1}-b_{2}+1-\gamma_{N}
$$


hold.

(ii) In the case $\alpha=(1,-1)$ the estimates

$$
a_{1}^{h} \leq a_{1}-n_{2}-2 b_{1}+1-\gamma_{N}, \quad a^{h} \leq a-n_{2}-2 b_{1}+1-\gamma_{N}
$$

hold.

(iii) In the case $\alpha=(0,0)$ the estimates

$$
a_{1}^{h} \leq a_{1}-n_{2}-b_{1}-\gamma_{N}, \quad a^{h} \leq a-n_{2}-b_{1}-\gamma_{N}
$$

hold.

Proof is left to the reader.

To conclude, we remind the reader that when a quadratic factor is being split off, the ambient space for the subvariety $B^{h}$ is $\mathcal{P}_{N-1}^{\left(n_{1}-1, n_{2}\right)}$, and the codimension $a^{h}$ is the codimension of the subvariety $B_{h}$ with respect to the latter space.

3.3. The main inductive estimate. Let us come back to the main problem of estimating the multiplicities $\mu_{l, N}(B, d)$ for an irreducible bi-invariant subvariety $B \subset \mathcal{P}_{N}^{\left(n_{1}, n_{2}\right)}$ of codimension $a \geq 1$. Let $\tau(B)=\left(\left(a_{1}, b_{1}\right),\left(a_{2}, b_{2}\right)\right)$ be the type of the subvariety $B$, where $b=b_{1}+b_{2} \geq 1$ (otherwise by Proposition 2.2 there is nothing to estimate). The following key fact makes it possible to estimate the multiplicity $\mu_{l, N}$ from above in terms of similar multiplicities for $\mathbb{P}^{N-1}$, which gives an inductive (in $N$ ) procedure of estimating multiplicities.

Proposition 3.3. (i) Assume that $b_{2} \geq 1$. Then there exist non-zero linear forms $h_{1}, h_{2} \in \mathcal{P}_{1, N}$, depending on the variety $B$ only and a set of non-negative integers

$$
\left(\begin{array}{ll}
d_{11} & d_{12} \\
d_{21} & d_{22}
\end{array}\right) \in \operatorname{Mat}_{2,2}\left(\mathbb{Z}_{+}\right),
$$

satisfying the equalities $d_{11}+d_{12}=d_{21}+d_{22}=d$, such that the subvarieties

$$
B_{1}=B_{h_{1}} \subset \mathcal{P}_{N-1}^{\left(n_{1}, n_{2}-1\right)} \quad \text { and } \quad B_{2}=B_{h_{2}}\left(h_{1} h_{2}\right) \subset \mathcal{P}_{N-1}^{\left(n_{1}, n_{2}-1\right)}
$$

satisfy the inequality

$$
\begin{aligned}
\mu_{l, N}(B, d) \leq & \mu_{l, N-1}\left(B_{1}, d_{11}\right)+\mu_{l-1, N-1}\left(B_{1}, d_{12}\right)+ \\
& +\mu_{l, N-1}\left(B_{2}, d_{21}\right)+\mu_{l-1, N-1}\left(B_{2}, d_{22}\right) .
\end{aligned}
$$

Moreover, the subvariety $B_{1}$ is of the type

$$
\tau\left(B_{1}\right)=\left(\left(a_{11}, b_{11}\right),\left(a_{12}, b_{12}\right)\right) \quad \text { with } \quad\left(b_{11}, b_{12}\right)=\left(b_{1}, b_{2}-1\right) .
$$

(ii) Assume that $b_{1} \geq 1$. Then there exist non-zero linear forms $h_{1}, h_{2}$, depending on $B$ only, and a set of non-negative integers $\left(d_{i j}\right)_{1 \leq i, j \leq 2}$, satisfying the equalities $d_{11}+d_{12}=d_{21}+d_{22}=d$, such that for the subvarieties

$$
B_{1}=B_{h_{1}} \subset \mathcal{P}_{N-1}^{\left(n_{1}-1, n_{2}\right)} \quad \text { and } \quad B_{2}=B_{h_{2}}\left(h_{1} h_{2}\right) \subset \mathcal{P}_{N-1}^{\left(n_{1}-1, n_{2}\right)}
$$


the inequality (16) is satisfied. Moreover, the subvariety $B_{1}$ has the type $\tau\left(B_{1}\right)=$ $\left(\left(a_{11}, b_{11}\right),\left(a_{12}, b_{12}\right)\right)$ with $\left(b_{11}, b_{12}\right)=\left(b_{1}-1, b_{2}\right)$.

Proof. Let us consider the case (i) in full detail. The second case is considered in an absolutely similar way. The bi-invariant irreducible subvariety $B$ is fixed. Let $h_{1} \in \mathcal{P}_{1, N}$ be a linear form of general position, where the genericity is understood in the following sense: for a generic tuple $\left(f_{1}, \ldots, f_{N}\right) \in B$ the linear subspace

$$
\left\{d f_{1}(o)=\ldots=d f_{N}(o)=0\right\}
$$

(which is of dimension $b=b_{1}+b_{2}$ ) is not contained in the hyperplane $\left\{h_{1}=0\right\}$. Let

$$
\Pi=\left\{h_{1}\left(z_{*}\right) h\left(z_{*}\right) \mid h \in \mathcal{P}_{1, N}\right\} \subset \mathcal{P}_{2, N}
$$

be the linear space of reducible homogeneous quadratic polynomials, divisible by $h_{1}$. Note that $\mathcal{P}_{2, N} \subset \mathcal{P}_{[2,3], N}$, so that $\Pi$ can be considered as a linear subspace in $\mathcal{P}_{[2,3], N}$. Obviously, $\operatorname{dim} \Pi=N$. Set

$$
\mathcal{P}_{\Pi}=\mathcal{P}_{[1,2], N}^{n_{1}-b_{1}} \times \mathcal{P}_{2, N}^{b_{1}} \times \mathcal{P}_{[1,3], N}^{n_{2}-b_{2}} \times \mathcal{P}_{[2,3], N}^{b_{2}-1} \times \Pi
$$

As we explained above, $\mathcal{P}_{\Pi}$ is a closed irreducible subset of the space

$$
\mathcal{P}_{\Pi}=\mathcal{P}_{[1,2], N}^{n_{1}-b_{1}} \times \mathcal{P}_{2, N}^{b_{1}} \times \mathcal{P}_{[1,3], N}^{n_{2}-b_{2}} \times \mathcal{P}_{[2,3], N}^{b_{2}} .
$$

Lemma 3.1. (i) The intersection $\bar{B} \cap \mathcal{P}_{\Pi}$ is non-empty and its codimension in $\mathcal{P}_{\Pi}$ is not higher than $\operatorname{codim} \bar{B}$.

(ii) The closure $\overline{\pi_{2}\left(\bar{B} \cap \mathcal{P}_{\Pi}\right)}$ coincides with $[\bar{B}]_{N-1}$.

Proof. Let us show the claim (i). By the bi-invariance the closed set $\bar{B}$ contains the zero tuple $(0, \ldots, 0) \in \mathcal{P}_{N}^{\left(n_{1}, n_{2}\right)}$. Therefore $\bar{B} \cap \mathcal{P} \neq \emptyset$, and the rest is obvious.

Now let us show the claim (ii). Recall that $\pi_{2}$ is the projection along the last direct factor $\mathcal{P}_{[2,3], N}$. By the equality (12) the codimension of the set $\bar{B}$ is strictly smaller than $N$. Therefore, for a tuple of general position $\left(f_{1}, \ldots, f_{N-1}\right) \in[\bar{B}]_{N-1}$ the intersection of the fibre $[\bar{B}]^{N}\left(f_{1}, \ldots, f_{N-1}\right)$ with the subspace $\Pi$ in the space $\mathcal{P}_{[2,3], N}$ is non-empty and for that reason has a positive dimension. (The non-emptiness is again a consequence of being bi-invariant:

$$
\left.\left(f_{1}, \ldots, f_{N-1}, 0\right) \in \bar{B} .\right)
$$

This proves the claim (ii). Q.E.D. for the lemma.

Remark 3.1. Without loss of generality, we may assume that the irreducible subvariety $B$ is an irreducible component of the closed set $X_{l, N}(m, d)$.

The equality $\mu_{l, N}(B, d)=m$ means that for a generic tuple $\left(f_{1}, \ldots, f_{N}\right) \in \bar{B}$ and any effective cycle $R$ of codimension $l$ and degree $d$ the inequality $\mu_{R}\left(f_{1}, \ldots, f_{N}\right) \leq m$ holds, and moreover, for some cycle $R$ (depending on the tuple $\left(f_{*}\right)$ ) the equality holds. Therefore, for a generic tuple

$$
\left(f_{1}, \ldots, f_{N-1}, h_{1}\left(z_{*}\right) h_{2}\left(z_{*}\right)\right) \in \bar{B} \cap \mathcal{P}_{\Pi}
$$


there is en effective cycle $R$, depending on that tuple, for which the inequality

$$
\mu_{R}\left(f_{1}, \ldots, f_{N-1}, h_{1} h_{2}\right) \geq m
$$

is satisfied. The form $h_{2}\left(z_{*}\right)$, as we explained above, is non-zero. Let $H_{i}=\left\{h_{i}=\right.$ $0\}, i=1,2$, be the corresponding hyperplanes. Define the effective cycles $R_{i j}$ of codimension $l, i, j=1,2$, by the conditions:

- $R=R_{11}+R_{12}=R_{21}+R_{22}$,

- Supp $R_{12} \subset H_{1}$ and none of the irreducible components of the cycle $R_{11}$ is contained in $H_{1}$,

- Supp $R_{22} \subset H_{2}$ and none of the irreducible components of the cycle $R_{21}$ is contained in $\mathrm{H}_{2}$.

Set $d_{i j}=\operatorname{deg} R_{i j}$. Obviously, $d=d_{11}+d_{12}=d_{21}+d_{22}$. By what was said above, the inequality

$$
m \leq \mu_{R}\left(f_{1}, \ldots, f_{N-1}, h_{1}\right)+\mu_{R}\left(f_{1}, \ldots, f_{N-1}, h_{2}\right)
$$

holds, and for any $i \in\{1,2\}$

$$
\mu_{R}\left(f_{1}, \ldots, f_{N-1}, h_{i}\right) \leq \mu_{R_{i 1}}\left(f_{1}, \ldots, f_{N-1}, h_{i}\right)+\mu_{R_{i 2}}\left(f_{1}, \ldots, f_{N-1}, h_{i}\right) .
$$

Since the irreducible components of the cycle $R_{i 1}$ are not contained in the hyperplane $H_{i}$, and $\operatorname{Supp} R_{i 2} \subset H_{i}$, for the first and second multiplicities in the right hand side of the last inequality we get the estimates

$$
\mu_{R_{i 1}}\left(f_{1}, \ldots, f_{N-1}, h_{i}\right) \leq \mu_{\left(R_{i 1} \circ H_{i}\right)}\left(\left.f_{1}\right|_{H_{i}}, \ldots,\left.f_{N-1}\right|_{H_{i}}\right)
$$

and

$$
\mu_{R_{i 2}}\left(f_{1}, \ldots, f_{N-1}, h_{i}\right) \leq \mu_{R_{i 2}}\left(\left.f_{1}\right|_{H_{i}}, \ldots,\left.f_{N-1}\right|_{H_{i}}\right),
$$

respectively. Note that $\left(R_{i 1} \circ H_{i}\right)$ is an effective cycle of codimension $l$ and degree $d_{i 1}$ on $H_{i} \cong \mathbb{P}^{N-1}$, and $R_{i 2}$ is an effective cycle of codimension $l-1$ on $H_{i} \cong \mathbb{P}^{N-1}$. Furthermore,

$$
\left(\left.f_{1}\right|_{H_{1}}, \ldots,\left.f_{N-1}\right|_{H_{1}}\right) \in \pi_{h_{1}}\left([\bar{B}]_{N-1}\right)
$$

and

$$
\left(\left.f_{1}\right|_{H_{2}}, \ldots,\left.f_{N-1}\right|_{H_{2}}\right) \in \pi_{h_{2}}\left(\bar{B}\left(h_{1} h_{2}\right)\right)
$$

are generic tuples, so that the inequalities (19) and (20) remain true, if in the right hand side that tuple is replaced by a generic tuple of polynomials

$$
\left(g_{1}, \ldots, g_{N-1}\right) \in B_{i} .
$$

Now the inequality (16) is a direct corollary of (17), (18), (19) and (20)). 
Finally, by the genericity of the form $h_{1}$ we have

$$
\operatorname{rk}\left(d g_{1}(o), \ldots, d g_{N-1}(o)\right)=N-b,
$$

as it was claimed. Q.E.D. for Proposition 3.3.

In order to make our inductive procedure a working one, it remains to estimate the codimension of the subvarieties $B_{2}$ and their type. Again we consider more closely the case of the projection onto the last factor (that is, $\left.\left(f_{*}\right) \mapsto f_{N}\right)$, corresponding to the part (i) of Proposition 3.3. In the case of the projection onto the last quadratic factor (that is, $\left.\left(f_{*}\right) \mapsto f_{n_{1}}\right)$ the modifications are obvious and we give only the final result. Set $\gamma=\gamma\left(h_{1} h_{2}\right)$ (see Sec. 3.1).

Proposition 3.4. Let $h_{2} \in \mathcal{P}_{1, N}$ be a generic linear form such that $h_{1} h_{2} \in$ $\lambda_{2}\left(\bar{B} \cap \mathcal{P}_{\Pi}\right)$. There exists and integer-valued vector

$$
\alpha=\left(\alpha_{1}, \alpha_{2}\right) \in\{(0,1),(-1,1),(0,0)\}
$$

such that for the parameters $\left(\bar{b}_{1}, \bar{b}_{2}\right)$ of the subvariety $B_{h_{2}}\left(h_{1} h_{2}\right)$ the following equalities hold:

$$
\bar{b}_{1}=b_{1}-\alpha_{1}, \quad \bar{b}_{2}=b_{2}-\alpha_{2} .
$$

For the codimension $\bar{a}$ of the subvariety $B_{h_{2}}\left(h_{1} h_{2}\right)$ in the ambient space $\mathcal{P}_{N-1}^{\left(n_{1}, n_{2}-1\right)}$ the following estimates hold.

(i) In the case $\alpha=(0,1): \bar{a} \leq a-(2 b-1)-\gamma$.

(ii) In the case $\alpha=(-1,1) \bar{a} \leq a-b-\gamma$.

(iii) In the case $\alpha=(0,0): \bar{a} \leq a-b_{1}-b-\gamma$.

Proof is almost word for word the same as that of Proposition 3.1. Although the set $\bar{B}\left(h_{1} h_{2}\right)$ may be not invariant under the linear changes of coordinates, it is still invariant under the operations of taking linear combinations, in the same way as the set $[\bar{B}]_{N-1}$. By Lemma 3.1,

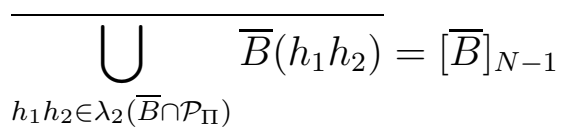

and for this reason for a generic tuple $\left(g_{1}, \ldots, g_{N-1}\right) \in \bar{B}\left(h_{1} h_{2}\right)$ the linear forms

$$
d g_{1}(o), \ldots, d g_{n_{1}-b_{1}}(o), d g_{n_{1}+1}(o), \ldots, d g_{N-b_{2}}(o)
$$

are linearly independent. Taking this into account, the proof of Proposition 3.1 works word for word, given the inequality (14), and with simplifications as we claim nothing about the parameter $\bar{a}_{1}$ of the full type of $B_{h_{2}}\left(h_{1} h_{2}\right)$. Q.E.D.

Now let us formulate the result for the case when a quadratic factor is split off.

Proposition 3.5. Let $h_{2} \in \mathcal{P}_{1, N}$ be a generic linear form such that $h_{1} h_{2} \in$ $\lambda_{1}\left(\bar{B} \cap \mathcal{P}_{\Pi}\right)$. There exists and integer-valued vector

$$
\alpha=\left(\alpha_{1}, \alpha_{2}\right) \in\{(1,0),(1,-1),(0,0)\}
$$


such that for the parameters $\left(\bar{b}_{1}, \bar{b}_{2}\right)$ of the subvariety $B_{h_{2}}\left(h_{1} h_{2}\right)$ the following equalities hold:

$$
\bar{b}_{1}=b_{1}-\alpha_{1}, \quad \bar{b}_{2}=b_{2}-\alpha_{2} .
$$

For the codimension $\bar{a}$ of the subvariety $B_{h_{2}}\left(h_{1} h_{2}\right)$ in the ambient space $\mathcal{P}_{N-1}^{\left(n_{1}-1, n_{2}\right)}$ the following estimates hold.

(i) In the case $\alpha=(1,0): \bar{a} \leq a-n_{2}-2 b_{1}-b_{2}+1-\gamma$.

(ii) In the case $\alpha=(1,-1) \bar{a} \leq a-n_{2}-2 b_{1}+1-\gamma$.

(iii) In the case $\alpha=(0,0): \bar{a} \leq a-n_{2}-b_{1}-\gamma$.

Proof is almost word for word the same as that of Proposition 3.2, and follows the same procedure as was used in the proof of Proposition 3.1. Q.E.D.

\section{§4. Local multiplicities. III. Explicit estimates}

In this section, using the inductive procedure, developed in $\S 3$, we obtain explicit estimates for the local multiplicity. We consider separately the cases of small values $b=1,2$ and small codimensions $a \leq 36$. We prove Theorems 4 and 5 .

4.1. An estimate for the multiplicity in the case $b=b_{1}+b_{2}=1$. We did see above that for an irreducible subvariety $B \subset \mathcal{P}_{N}^{\left(n_{1}, n_{2}\right)}$ with $b=0$ the equality $\mu_{l, N}(B, d)=d$ holds (Proposition 2.2). Let us consider the case $b=1$, the next in complexity. Assume for certainty that $b_{2}=1, b_{1}=0$. By Proposition 3.3, the inequality

$$
\mu_{l, N}(B, d) \leq d+\mu_{l, N-1}\left(B_{2}, d_{21}\right)+\mu_{l-1, N-1}\left(B_{2}, d_{22}\right)
$$

holds, since $\left(b_{11}, b_{12}\right)=(0,0)$. Let $\tau\left(B_{2}\right)=\left(\left(a_{21}, b_{21}\right),\left(a_{22}, b_{22}\right)\right)$ be the type of the variety $B_{2}$. If $b_{21}=b_{22}=0$, then we get the estimate $\mu_{l, N}(B, d) \leq 2 d$. Assume that $b_{21}+b_{22}=1$. Setting $B_{2}=B^{(1)}$, let us apply Proposition 3.3 to that subvariety. Iterating this construction, we obtain a chain of subvarieties

$$
B^{(1)}, \ldots, B^{(k)}
$$

of the type $\tau\left(B^{(i)}\right)=\left(\left(a_{1}^{(i)}, b_{1}^{(i)}\right),\left(a_{2}^{(i)}, b_{2}^{(i)}\right)\right)$ with $b^{(i)}=b_{1}^{(i)}+b_{2}^{(i)}=1$. Here $B^{(i+1)}=$ $B_{2}^{(i)}$ in the sense of Proposition 3.3. The varieties $B^{(i)}$ are irreducible bi-invariant subvarieties of the space $\mathcal{P}_{N-i}^{\left(n_{1}^{(i)}, n_{2}^{(i)}\right)}$, the corresponding subvariety $B_{1}^{(i)}$ has the type $\left(\left(a_{11}^{(i)}, 0\right),\left(a_{12}^{(i)}, 0\right)\right)$ and its input into the estimate of the multiplicity $\mu_{l, N}$ is known. After $k$ steps we get the inequality неравенство

$$
\mu_{l, N}(B, d) \leq k d+\sum_{j=0}^{\min \{k, l\}} \mu_{l-j, N-k}\left(B^{(k)}, d_{k, j}\right),
$$

where $d_{k, 0}+\ldots+d_{k, \min \{k, l\}}=d$. We used the obvious inequality

$$
\mu_{l, N}\left(B, d^{\prime}\right)+\mu_{l, N}\left(B, d^{\prime \prime}\right) \leq \mu_{l, N}\left(B, d^{\prime}+d^{\prime \prime}\right) .
$$


By Propositions 3.1 and 3.2 for the codimension $a^{(i)}=\operatorname{codim} B^{(i)}$ we have the inequality $a^{(i+1)} \leq a^{(i)}-1$, and for the variety $B^{(i)}$ to exist, the inequality

$$
a^{(i)}-b_{1}^{(i)}\left(k_{2}^{(i)}+b_{1}^{(i)}\right)-b_{2}^{(i)} b^{(i)} \geq 0
$$

should be satisfied. Therefore, $a \geq a^{(k)}+k \geq k+1$, so that after $k \leq a-1$ steps we get $b_{21}^{(k)}=b_{22}^{(k)}=0$ and the procedure is completed. As a result we obtain the inequality

$$
\mu_{l, N}(B, d) \leq(a+1) d .
$$

Note that for $l=0$ (when all effective cycles are of the form $d \mathbb{P}^{N}$ ) this estimate is precise: the equalities $b_{1}=0, b_{2}=1$ mean that for a generic tuple $\left(f_{1}, \ldots, f_{N}\right) \in B$ the complete intersection

$$
\left\{f_{1}=\ldots=f_{N-1}=0\right\}
$$

is a curve, non-singular at the point $o$. The condition of tangency of order $j \leq N$ with that curve imposes at most $j$ independent conditions on the polynomial $f_{N}$. Therefore, the equality

$$
\mu_{0, N}(a, d)=(a+1) d
$$

holds.

4.2. Estimating the multiplicity in the case $b=2$. Similarly to Sec. 2.2 , set $\mu_{l, N}(a, b ; d)=m$, if for any irreducible (bi-invariant) subvariety $B \subset \mathcal{P}_{N}^{\left(n_{1}, n_{2}\right)}$ of codimension at most $a$ with $\varepsilon(B)=b$ the inequality $\mu_{l, N}(B, d) \leq m$ holds, and for at least one subvariety $B$ in that class the inequality is an equality. The result of Sec. 4.1 can be represented as the inequality

$$
\mu_{l, N}(a, 1 ; d) \leq(a+1) d
$$

Now let us obtain an upper bound for $\mu_{l, N}(a, 2 ; d)$. Let $B$ be a subvariety with $\varepsilon(B)=2$. Applying the result of Sec. 4.1, we get

$$
\mu_{l, N}(B, d) \leq(a-2) d+\mu_{l, N-1}\left(B_{2}, d_{21}\right)+\mu_{l-1, N-1}\left(B_{2}, d_{22}\right)
$$

for some $d_{21}, d_{22} \in \mathbb{Z}_{+}$with $d_{21}+d_{22}=d$. For the parameters of the subvariety $B_{2}$ there are two options:

- either $\operatorname{codim} B_{2} \leq a-2$ and $\varepsilon\left(B_{2}\right)=2$,

- or $\operatorname{codim} B_{2} \leq a-3$ and $\varepsilon\left(B_{2}\right)=1$.

In the second case we get the estimate

$$
\mu_{l, N}(B, d) \leq 2(a-2) d
$$

In the first case one can go on with the process of reduction, applying Proposition 3.3 to $B_{2}=B^{(1)}$. Arguing as in Sec. 4.1 (the computations are absolutely elementary 
and similar to those performed in Sec. 4.1 and we do not give them here), we obtain the following final result: for an even $a=2 u$ the inequality

$$
\mu_{l, N}(a, 2 ; d) \leq(2+u(u-1)) d
$$

holds, for an odd $a=2 u+1$ the inequality

$$
\mu_{l, N}(a, 2 ; d) \leq\left(2+u^{2}\right) d
$$

holds.

This procedure of obtaining explicit upper bounds for the numbers $\mu_{l, N}(a, b ; d)$ can be iterated, reducing the estimate for $b=3$ to the already known formulas for $b=1,2$. However, as could already see in the case $b=2$, the number of cases that require separate consideration, starts to grow, and the formulas get clumsier. Thus for small codimensions it is easier to obtain a particular numerical value of the upper bound for $\mu_{l, N}(a, b ; d)$, whereas for higher values of $a$ we need a less precise but manageable estimate.

4.3. Small codimensions. A simple observation that one can make on the basis of the considerations for $b=1$ and $b=2$, is the linearity of the obtained estimates in the degree $d$ and their actual independence of the parameters $l, N$. Let $U \subset \mathbb{Z}_{+} \times \mathbb{Z}_{+}$ be the set $\left\{(a, b) \mid a \geq b^{2}\right\}$. Let us define by induction the function

$$
\bar{\mu}: U \rightarrow \mathbb{Z}_{+},
$$

setting $\bar{\mu}(a, 0) \equiv 1, \bar{\mu}(a, 1) \equiv a+1$, for $a<b(b+1)$

$$
\bar{\mu}(a, b)=2 \bar{\mu}(a-(2 b-1), b-1),
$$

for $a \geq b(b+1)$

$$
\bar{\mu}(a, b)=\bar{\mu}(a-(2 b-1), b-1)+\max \{\bar{\mu}(a-(2 b-1), b-1), \bar{\mu}(a-b, b)\} .
$$

Propositions 3.1, 3.2 and 3.3 imply immediately

Proposition 4.1. The following inequality holds:

$$
\mu_{l, N}(a, b ; d) \leq \bar{\mu}(a, b) d
$$

For small values of $a$ the function $\bar{\mu}$ is easy to compute by hand; it is also easy to write a computer program, computing $\bar{\mu}$. Below we give the table of values $\bar{\mu}(a, b)$ for $a \leq 36, b \leq 6$. The symbol $*$ means that the pait $(a, b) \notin U$ and the value of the function $\bar{\mu}$ is not defined. Already for those small values of the codimension the speed of growth of the values $\bar{\mu}(a, b)$ can be seen very well. In boldface we show the maximum value $\bar{\mu}(a, b)$ for the given $a$. 


\begin{tabular}{|l|c|c|c|c|c|c|c|c|c|c|c|c|c|c|c|}
\hline$a$ & 1 & 2 & 3 & 4 & 5 & 6 & 7 & 8 & 9 & 10 & 11 & 12 & 13 & 14 & 15 \\
\hline$b=0$ & 1 & 1 & 1 & 1 & 1 & 1 & 1 & 1 & 1 & 1 & 1 & 1 & 1 & 1 & 1 \\
\hline$b=1$ & $\mathbf{2}$ & $\mathbf{3}$ & $\mathbf{4}$ & $\mathbf{5}$ & $\mathbf{6}$ & 7 & 8 & 9 & 10 & 11 & 12 & 13 & 14 & 15 & 16 \\
\hline$b=2$ & $*$ & $*$ & $*$ & 4 & 6 & $\mathbf{8}$ & $\mathbf{1 1}$ & $\mathbf{1 4}$ & $\mathbf{1 8}$ & $\mathbf{2 2}$ & $\mathbf{2 7}$ & $\mathbf{3 2}$ & $\mathbf{3 8}$ & $\mathbf{4 4}$ & $\mathbf{5 1}$ \\
\hline$b=3$ & $*$ & $*$ & $*$ & $*$ & $*$ & $*$ & $*$ & $*$ & 8 & 12 & 16 & 22 & 28 & 36 & 44 \\
\hline$b=4$ & $*$ & $*$ & $*$ & $*$ & $*$ & $*$ & $*$ & $*$ & $*$ & $*$ & $*$ & $*$ & $*$ & $*$ & $*$ \\
\hline
\end{tabular}

\begin{tabular}{|l|c|c|c|c|c|c|c|c|c|c|c|}
\hline$a$ & 16 & 17 & 18 & 19 & 20 & 21 & 22 & 23 & 24 & 25 & 26 \\
\hline$b=0$ & 1 & 1 & 1 & 1 & 1 & 1 & 1 & 1 & 1 & 1 & 1 \\
\hline$b=1$ & 17 & 18 & 19 & 20 & 21 & 22 & 23 & 24 & 25 & 26 & 27 \\
\hline$b=2$ & $\mathbf{5 8}$ & 66 & 74 & 83 & 92 & 102 & 112 & 123 & 134 & 146 & 158 \\
\hline$b=3$ & 55 & $\mathbf{6 8}$ & $\mathbf{8 2}$ & $\mathbf{9 9}$ & $\mathbf{1 1 9}$ & $\mathbf{1 4 0}$ & $\mathbf{1 6 5}$ & $\mathbf{1 9 3}$ & $\mathbf{2 2 3}$ & $\mathbf{2 5 7}$ & $\mathbf{2 9 5}$ \\
\hline$b=4$ & 16 & 24 & 32 & 44 & 56 & 72 & 88 & 110 & 136 & 164 & 198 \\
\hline$b=5$ & $*$ & $*$ & $*$ & $*$ & $*$ & $*$ & $*$ & $*$ & $*$ & 32 & 48 \\
\hline$b=6$ & $*$ & $*$ & $*$ & $*$ & $*$ & $*$ & $*$ & $*$ & $*$ & $*$ & $*$ \\
\hline
\end{tabular}

\begin{tabular}{|l|c|c|c|c|c|c|c|c|c|c|}
\hline$a$ & 27 & 28 & 29 & 30 & 31 & 32 & 33 & 34 & 35 & 36 \\
\hline$b=0$ & 1 & 1 & 1 & 1 & 1 & 1 & 1 & 1 & 1 & 1 \\
\hline$b=1$ & 28 & 29 & 30 & 31 & 32 & 33 & 34 & 35 & 36 & 37 \\
\hline$b=2$ & 171 & 184 & 198 & 212 & 227 & 242 & 258 & 274 & 291 & 308 \\
\hline$b=3$ & $\mathbf{3 3 5}$ & $\mathbf{3 8 0}$ & $\mathbf{4 2 9}$ & $\mathbf{4 8 1}$ & $\mathbf{5 3 8}$ & $\mathbf{6 0 0}$ & $\mathbf{6 6 5}$ & $\mathbf{7 3 6}$ & 812 & 892 \\
\hline$b=4$ & 238 & 280 & 330 & 391 & 461 & 537 & 625 & 726 & $\mathbf{8 4 1}$ & $\mathbf{9 6 6}$ \\
\hline$b=5$ & 64 & 88 & 112 & 144 & 176 & 220 & 272 & 328 & 396 & 476 \\
\hline$b=6$ & $*$ & $*$ & $*$ & $*$ & $*$ & $*$ & $*$ & $*$ & $*$ & 64 \\
\hline$b=7$ & $*$ & $*$ & $*$ & $*$ & $*$ & $*$ & $*$ & $*$ & $*$ & $*$ \\
\hline
\end{tabular}

Now let us consider the problem of obtaining a simple effective upper bound for the multiplicities $\mu_{l, N}(B, d)$. From the technical viewpoint, it is necessary to find a simple and visual formalization of the procedure of estimating these numbers in terms of the numbers $\mu_{l^{\prime}, N^{\prime}}\left(B^{\prime}, d^{\prime}\right)$, where the varieties $B^{\prime} \subset \mathcal{P}_{N^{\prime}}^{\left(n_{1}^{\prime}, n_{2}^{\prime}\right)}$ have a smaller value $b^{\prime}=b_{1}^{\prime}+b_{2}^{\prime}$, so that for the corresponding multiplicities the upper bound can be assumed to be known. This gives an inductive (in the parameter $b$ ) procedure of estimating the multiplicity, realized below.

4.4. A general method of estimating the multiplicity. Let us consider the four-letter alphabet $\left\{A, C_{0}, C_{1}, C_{2}\right\}$. We define a procedure of constructing a certain set of words $W$ in this alphabet and for each word $w \in W$ a certain irreducible bi-invariant subvariety

$$
B[w] \subset \mathcal{P}_{N(w)}^{\left(n_{1}(w), n_{2}(w)\right)}
$$

of the type $\tau(B[w])=\left(\left(a_{1}(w), b_{1}(w)\right),\left(a_{2}(w), b_{2}(w)\right)\right.$ and full codimension $a(w)=$ $a_{1}(w)+a_{2}(w)$; as usual, set $\varepsilon(B[w])=b(w)=b_{1}(w)+b_{2}(w)$. The length of the word $w$ we denote by the symbol $|w| \in \mathbb{Z}_{+}$. The length of the empty word is equal to zero.

Let $B \subset \mathcal{P}_{N}^{\left(n_{1}, n_{2}\right)}$ be an irreducible bi-invariant variety of codimension $a$ and 
type $\tau(B)$. Set $B[\emptyset]=B$. If $b(\emptyset)=0$, then we set $W=\{\emptyset\}$ : the procedure is thus complete. Assume that $b(\emptyset) \geq 1$.

The set of words $W$ and corresponding subvarieties $B[w], w \in W$, will be constructed in elementary steps: we will construct a sequence of finite subsets $W_{l}$ of the set of all words, $l=0,1, \ldots$. The set $W_{0}=\{\emptyset\}$ is already constructed. Assume that $W_{0}, \ldots, W_{l}$ are constructed. If for every $w \in W_{l}$ the equality $b(w)=0$ holds, then we set $W=W_{l}$, completing the procedure. Otherwise, take any word $w \in W_{l}$ with $b(w) \geq 1$. If $b_{2}(w) \geq 1$, then we apply to the subvariety $B[w]$ (constructed at the previous step) part (i) of Proposition 3.3. Set $w_{1}=w A$ and $w_{2}=w C_{e}$, where $e \in\{0,1,2\}$ is chosen in the following way.

Set $B\left[w_{1}\right]=(B[w])_{1}$ and $B\left[w_{2}\right]=(B[w])_{2}$ in the notations of Proposition 3.3, (i). Now $e$ takes the value 0,1 or 2 , if the subvariety $B\left[w_{2}\right]$ corresponds to the case (i), (ii) or (iii) of Proposition 3.1, respectively. This determines the words $w_{1}$ and $w_{2}$. The set $W_{l+1}$ is obtained from $W_{l}$ by removing the word $w$ and adding the words $w_{1}, w_{2}$ of length $|w|+1$. The irreducible bi-invariant subvarieties $B\left[w_{i}\right]$ were constructed above, and this defines the values of all parameters $\left(n_{i}(w)\right.$ etc.).

If $b_{2}(w)=0$, then $b_{1}(w) \geq 1$ and we apply to the subvariety $B[w]$ part (ii) of Proposition 3.3. Now the words $w_{1}, w_{2}$ and the subvarieties $B\left[w_{i}\right]$ are constructed word for word in the same way as in the case $b_{2}(w) \geq 1$, replacing part (i) of Proposition 3.3 by part (ii) of the same proposition and Proposition 3.1 by Proposition 3.2.

Now the procedure of constructing the set $W_{l+1}$ is determined in a unique way. Obviously,

$$
\sharp W_{l}=l+1 .
$$

Since when we replace a word $w \in W_{l}$ by the words $w_{1}, w_{2}$, the codimension of the new subvarieties $B\left[w_{i}\right]$ gets strictly smaller than the codimension of $B[w]$ (Propositions 3.1 and 3.2), our procedure of constructing the sequence $\left\{W_{l}\right\}$ can not be infinite. It is easy to see that for $\operatorname{codim} B=a$ we get $\sharp W \leq 2^{a}$. Now Proposition 3.3 implies immediately

Proposition 4.2. The following inequality holds:

$$
\mu_{l, N}(B, d) \leq d(\sharp W) .
$$

Proof. This follows from a more general fact:

$$
\mu_{l, N}(B, d) \leq \sum_{w \in W_{i}} \sum_{j=0}^{\min \{l,|w|\}} \mu_{l-j, N-|w|}\left(B[w], d_{j}(w)\right)
$$

for any $i=0,1, \ldots$ and certain partitions

$$
d=\sum_{j=0}^{\min \{l,|w|\}} d_{j}(w)
$$


for every word $w \in W_{i}$. The inequality (22) will be shown by induction on the parameter $i=0,1, \ldots$ For $i=0$ its left hand side and right hand side are the same. Assume that (22) is shown for $i=0, \ldots, e$. If $W_{e}=W_{e+1}$, then $W_{e}=W$ and there is nothing more to prove. If $W_{e} \neq W_{e+1}$, then the set of words $W_{e+1}$ is obtained from $W_{e}$ by removing some word $w \in W_{e}$ and ading two words $w_{1}, w_{2}$. Therefore, in order to prove the inequality (22) for $i=e+1$ it is sufficient to show that

$$
\sum_{j=0}^{\min \{l,|w|\}} \mu_{l-j, N-|w|}\left(B[w], d_{j}[w]\right)
$$

does not exceed the sum of similar expressions for $w_{1}, w_{2}$. This is precisely what Proposition 3.3 claims, taking into account the inequality (21). This proves the estimate (22) for any $i$. Finally, if $W_{i}=W$, then for any $w \in W$ we have $b(w)=0$, so that in the right hand side of the inequality (22) for each component we have the equality

$$
\mu_{l-j, N-|w|}\left(B[w], d_{j}(w)\right)=d_{j}(w),
$$

so that by the equality (23) we get

$$
\mu_{l, N}(B, d) \leq d \sum_{w \in W} 1
$$

which is what we claimed. Q.E.D. for Proposition 4.2.

4.5. Estimating the cardinality of the set of words. We write down the words in the following way:

$$
w=\tau_{1} \ldots \tau_{K}
$$

where $\tau_{i} \in\left\{A, C_{0}, C_{1}, C_{2}\right\}$. Now let

$$
\nu:\left\{A, C_{0}, C_{1}, C_{2}\right\} \rightarrow\{A, C\}
$$

be the map of the four-letter alphabet into the two-letter one, given by the equalities $\nu(A)=A, \nu\left(C_{i}\right)=C$, and

$$
\nu: w=\tau_{1} \ldots \tau_{K} \mapsto \bar{w}=\nu\left(\tau_{1}\right) \ldots \nu\left(\tau_{K}\right)
$$

the corresponding map of the set of words. The following fact is true.

Lemma 4.1. For any $i=0,1, \ldots$ the map $\left.\nu\right|_{W_{i}}$ is injective. In particular, $\left.\nu\right|_{W}$ is injective.

Proof. A stronger fact is true: no word among the words $\bar{w}=\nu(w), w \in W_{i}$, is a left segment of any other word in this set. (In particular, no two words are the same, which means precisely the injectivity of the map $\left.\nu\right|_{W_{i}}$.) The last claim is easy to show by induction. The set $W_{0}$ consists of one word, and for this set the claim is trivial. Assume that it is shown for $W_{i}$, where $i=0, \ldots, e$. If $W_{e+1}=W_{e}$, then there is nothing to prove. If $W_{e+1} \neq W_{e}$, then $W_{e+1}$ is obtained from $W_{e}$ by removing some word $w \in W_{e}$ and adding two words $w_{1}=w A$ and $w_{2}=w C_{\alpha}$, where $\alpha \in\{0,1,2\}$. 
For these words we have $\bar{w}_{1}=\bar{w} A$ and $\bar{w}_{2}=\bar{w} C$. Obviously, $\bar{w}_{1}$ and $\bar{w}_{2}$ are not left segments of each other and no word $\bar{w}_{1}^{\prime}$ for $w^{\prime} \in W_{e} \backslash\{w\}$ is a left segment of $\bar{w}_{1}$ or $\bar{w}_{2}$, as otherwise $\bar{w}^{\prime}=\bar{w}_{1}$ or $\bar{w}_{2}$ (since $\bar{w}^{\prime}$ is not a left segment of the word $\bar{w}$ by the induction hypothesis), but then $\bar{w}$ would be a left segment of the word $\bar{w}^{\prime}$, contrary to the induction hypothesis. In the trivial way $\bar{w}_{1}$ and $\bar{w}_{2}$ are no left segments of any word $\bar{w}^{\prime}$, since otherwise this would have been true for $\bar{w}$ as well, contrary to the induction hypothesis. Q.E.D. for the lemma.

Thus we have reduced the problem of estimating the multiplicity $\mu_{l, N}(B, d)$ to the problem of estimating the number of words in the set $W$. As we pointed out above, $\sharp W \leq 2^{a}$, but that estimate is too coarse for our purposes. We will control the length of words $w \in W$ via the values of the parameters $a\left(w^{\prime}\right)$ and $b\left(w^{\prime}\right)=\varepsilon(B[w])$ for the left segments $w^{\prime}$ of the word $w$.

Lemma 4.2. (i) If $\tau=A$ or $C_{0}$, then the inequality

$$
a\left(w^{\prime} \tau\right) \leq a\left(w^{\prime}\right)-\left(2 b\left(w^{\prime}\right)-1\right)
$$

holds and $b\left(w^{\prime} \tau\right)=b\left(w^{\prime}\right)-1$.

(ii) If $\tau=C_{1}$ or $C_{2}$, then the inequality

$$
a\left(w^{\prime} \tau\right) \leq a\left(w^{\prime}\right)-b\left(w^{\prime}\right)
$$

holds and $b\left(w^{\prime} \tau\right)=b\left(w^{\prime}\right)$.

Proof. This follows immediately from the inequalities of Propositions 3.1 and 3.2 , taking into account the obvious estimate $b_{i} \leq n_{i}, i=1,2$. Q.E.D. for the lemma.

Furthermore, the inequality (13) implies the estimate

$$
a(w) \geq b_{1}(w)\left(n_{2}(w)+b_{1}(w)\right)+b_{2}(w) b(w) \geq b^{2}(w)
$$

for every word $w$, in particular, for every word, which is a left segment of any word in $W$.

Example 4.1. In terms of the formalism, developed above, let us again consider the case $b=b(\emptyset)=1$. Here for any word $w \in W_{i}$ we have the alternative: either $b(w)=0$ (and in that case $w \in W)$, or $b(w)=1$ (and in that case $a(w \tau) \leq a(w)-1$ for any letter $\tau$ ), so that the set $W$ is of the form

$$
A, C_{i_{1}} A, C_{i_{1}} C_{i_{2}} A, \ldots, C_{i_{1}} C_{i_{2}} \ldots C_{i_{k}} A, C_{i_{1}} \ldots C_{i_{k}} C_{0},
$$

where $i_{\alpha} \in\{1,2\}$ and $k+1 \leq a$. Therefore, $\sharp W \leq a+1$, as we claimed above in Sec. 4.1.

Let us come back to the general case.

Proposition 4.3. The following inequality holds:

$$
\sharp W \leq 2^{b} \frac{a^{b}}{(b !)^{2}} .
$$


Proof. For any word $w \in W$ by construction $b(w)=0$. Since the letters $C_{1}, C_{2}$ do not change the value of the parameter $b$, whereas the letters $A$ and $C_{0}$ decrease it by 1 , we can conclude that in the word $w$ there are precisely $b$ positions, occupied by the letters $A$ and $C_{0}$. Let them be the positions with the numbers

$$
m_{1}+1, m_{1}+m_{2}+2, \ldots, m_{1}+m_{2}+\ldots+m_{b}+b,
$$

$m_{i} \in \mathbb{Z}_{+}$. By Lemma 4.2, we get the inequality

$$
\begin{aligned}
0 \leq a(w) \leq a & -m_{1} b & & -(2 b-1)- \\
& -m_{2}(b-1) & & (2(b-1)-1)- \\
& \ldots & & \\
& -m_{i}(b-(i-1)) & & (2(b-(i-1))-1)- \\
& \cdots & & \\
& m_{b} & & =a-b^{2}-\sum_{i=1}^{b} m_{i}(b-(i-1)),
\end{aligned}
$$

so that $\left(m_{1}, \ldots, m_{b}\right)$ is an arbitrary integer-valued point in the polytope

$$
\Delta=\left\{x_{1} \geq 0, \ldots, x_{b} \geq 0, b x_{1}+(b-1) x_{2}+\ldots+x_{b} \leq a-b^{2}\right\} \subset \mathbb{R}^{b} .
$$

Thus even if we assume that all possible distributions of the letters $A$ and $C_{0}$ on the selected positions are realized by the words $w \in W$ (in fact, that is not true: there are much fewer words in $W$, see Remark 4.1), then the following inequality holds:

$$
\sharp W \leq 2^{b} \cdot \sharp\left(\Delta \cap \mathbb{Z}^{b}\right) .
$$

Now let us estimate the number of integer-valued points in $\Delta$. In order to do that, consider a larger polytope

$$
\Delta^{+}=\left\{x_{1} \geq 0, \ldots, x_{b} \geq 0, b x_{1}+\ldots+x_{b} \leq a-\frac{b(b-1)}{2}\right\} \subset \mathbb{R}^{b} .
$$

Obviously, $\Delta \subset \Delta^{+}$.

Lemma 4.3. The following inequality holds:

$$
\sharp\left(\Delta \cap \mathbb{Z}^{b}\right) \leq \operatorname{vol}\left(\Delta^{+}\right) .
$$

Proof. With each point $x=\left(x_{1}, \ldots, x_{b}\right) \in \mathbb{R}^{b}$ we associate the unit cube

$$
\Gamma(x)=\left[x_{1}, x_{1}+1\right] \times\left[x_{2}, x_{2}+1\right] \times \ldots \times\left[x_{b}, x_{b}+1\right] \subset \mathbb{R}^{b},
$$

the vertex of which with the least value of the sum of coordinates $x_{1}+\ldots+x_{b}$ is the point $x$. If $x \in \Delta$, then $\Gamma(x) \subset \Delta^{+}$, since

$$
b+(b-1)+\ldots+1+a-b^{2}=a-\frac{b(b-1)}{2} .
$$


Therefore,

$$
\sharp\left(\Delta \cap \mathbb{Z}^{b}\right)=\sum_{x \in \Delta \cap \mathbb{Z}^{b}} \operatorname{vol}(\Gamma(x))=\operatorname{vol}\left(\bigcup_{x \in \Delta \cap \mathbb{Z}^{b}} \Gamma(x)\right) \leq \operatorname{vol}\left(\Delta^{+}\right),
$$

as we claimed. Q.E.D. for the lemma.

Computing the volume of the polytope $\Delta^{+}$and applying the Stirling formula, we get the estimate

$$
\sharp W \leq 2^{b} \frac{\left(a-\frac{b(b-1)}{2}\right)^{b}}{(b !)^{2}}=\frac{1}{2 \pi b e^{\theta / 6 b}}\left(\frac{2 a-b(b-1)}{b^{2}} e^{2}\right)^{b}
$$

for some $0<\theta<1$ (here $e$ is the base of the natural logarithm), so that the more so,

$$
\sharp W \leq u_{b}=\frac{1}{2 \pi b}\left(\frac{2 a-b(b-1)}{b^{2}} e^{2}\right)^{b} .
$$

Recall that $b \in\{1, \ldots,[\sqrt{a}]\}$. To obtain an effective bound for the number $\sharp W$ let us study the behaviour of the sequence $u_{b}$ for those values of $b$.

Lemma 4.4. The sequence $u_{b}$ is increasing, provided that the following inequality holds:

$$
2 a-b(b-1) \geq \frac{5}{2} b^{2}
$$

Proof. Write down

$$
\frac{u_{b+1}}{u_{b}}=\frac{1}{1+\frac{1}{b}} \frac{e^{2}}{\left(1+\frac{1}{b}\right)^{2 b}} \frac{1}{\left(1+\frac{2 b}{2 a-b(b+1)}\right)^{b}} \frac{2 a-b(b+1)}{(b+1)^{2}} .
$$

Assume first that $b \geq 9$. If the numbers $a$ and $b$ satisfy the inequality $2 a-b(b+1) \geq$ $\frac{5}{2}(b+1)^{2}$ (that is, the inequality (26) for $b+1$ ), then the denominator of the third factor in the right hand side can be estimated from above as follows:

$$
\left(1+\frac{2 b}{2 a-b(b+1)}\right)^{b} \leq\left(1+\frac{4}{5} \frac{1}{b}\right)^{b}<e^{\frac{4}{5}} .
$$

The second factor in the right hand side of the inequality (27) is strictly higher than one, and the fourth is not smaller than $\frac{5}{2}$. As a result we get:

$$
\frac{u_{b+1}}{u_{b}}>\frac{9}{10} \cdot \frac{5}{2} \cdot e^{-\frac{4}{5}}>1
$$

which is what we need. For the smaller values $b \leq 8$ the second and third factors in the right hand side of the inequality (27) could be estimated more precisely, and elementary computations with some use of a computer complete the proof of the lemma. 
Corollary 4.1. For $a \geq 17$ the value $b_{\max } \in\{1, \ldots,[\sqrt{a}]\}$, on which the maximum of the sequence $u_{b}$ is attained, satisfies the inequality

$$
2 a-b_{\max }\left(b_{\max }-1\right) \leq \frac{5}{3} a .
$$

Proof. By the previous lemma, the value $b_{\max }$ satisfies the inequality

$$
2 a-b_{\max }\left(b_{\max }+1\right) \leq \frac{5}{2} b_{\max }^{2}
$$

(otherwise, the next element of the sequence $u_{b}$ would be higher). Now elementary computations complete the proof of the corollary.

Corollary 4.2. (i) For $a \geq 17$ the following estimate holds:

$$
\sharp W \leq v_{b}=\frac{1}{2 \pi b}\left(\frac{5 a}{3 b^{2}} e^{2}\right)^{b} .
$$

(ii) For any a the following estimate holds:

$$
\sharp W \leq w_{b}=\frac{1}{2 \pi b}\left(\frac{2 a}{b^{2}} e^{2}\right)^{b} .
$$

Proof. The claim (ii) follows immediately from the inequality (25), the claim (i) from the inequality (25), taking into account the previous corollary.

Corollary 4.3. (i) For $a \geq 17$ the following estimate holds:

$$
\mu_{l, N}(a, d) \leq \frac{e^{2}}{2 \pi[\sqrt{a}]}\left(\frac{5}{3} e^{2}\right)^{[\sqrt{a}]} .
$$

(ii) For any a the following estimate holds:

$$
\mu_{l, N}(a, d) \leq \frac{e^{2}}{2 \pi[\sqrt{a}]}\left(2 e^{2}\right)^{[\sqrt{a}]} .
$$

Proof. The arguments are identical in both cases, the only difference is which of the two claims of Corollary 4.2 is used.

Let us prove part (i). Arguing as in the proof of Lemma 4.4, we conclude that the sequence $v_{b}$ is increasing. Therefore, its maximum is attained at $b=[\sqrt{a}]$. Since

$$
a<(b+1)^{2}=b^{2}+2 b+1,
$$

we get the inequality

$$
\left(\frac{a}{b^{2}}\right)^{b} \leq\left(1+\frac{2}{b}\right)^{b}<e^{2},
$$


whence immediately follows the claim (i). The second part is shown in word for word the same way. Q.E.D.

It is easy to see that the claims of Theorems 4 and 5 are contained in the claims of Corollaries 4.2 and 4.3, taking into account the formula (25).

Q.E.D. for Theorems 4 and 5.

Remark 4.1. As we see from the given proof, the estimate obtained above is not optimal and can be essentially improved. For $b \approx \sqrt{a}$ we have $2 a-b(b-1) \approx a$, so that in the inequality of Corollary 4.3 , (ii), the expression $\left(2 e^{2}\right)$ can be replaced by $e^{2}$. Furthermore, when proving Proposition 4.3 , we took into account all possible tuples of positions $\left(m_{1}, \ldots, m_{b}\right)$ and all possible ways of putting the letters $A$ and $C_{0}$ on the $b$ positions. However, since in the set of words $\bar{W}=\nu(W)$ in the two-letter alphabet $\{A, C\}$ no word is a left segment of another word and the map $\nu: W \rightarrow \bar{W}$ is one-to-one, for a fixed way of putting the letters $A$ and $C_{0}$ on $b$ positions, when at least two letters $C_{0}$ are neighbours, not all tuples $\left(m_{1}, \ldots, m_{b}\right) \in \Delta \cap \mathbb{Z}^{b}$ are realized, as two distinct words $w_{1} \neq w_{2},\left\{w_{1}, w_{2}\right\} \subset W$, can not differ only on a segment that consists of the letters $C_{0}, C_{1}, C_{2}$. The problem of getting the precise upper bound for the numbers $\mu_{l, N}(a, d)$, at least in the asymptotic sense, remains open.

\section{§5. Global multiplicities. Proof of Theorem 2.}

In this section, using the theory developed in $\S \S 2-4$, we prove Theorem 2 . Taking into account Theorem 2 in the paper [3], two facts require a proof: the linear independence of the directions the lines passing through the point $o \in V$ (the last requirement in the condition (R1)), and that the condition (R3) is satisfied at every point $o \in V$ on a Zarisky generic complete intersection $V$ of the type $2^{k_{1}} 3^{k_{2}}$. It is not hard to prove the linear independence: it is sufficient to estimate the codimension of the sets of tuples of polynomials, which either have a positive-dimensional set of solutions or a finite set set of linearly dependent solutions. This is done in Sec. 5.1.

In Sec. 5.2-5.4 we globalize the constructions and results of the local theory, developed in $\S \S 2-4$ : define the global multiplicities, reduce the problem of their estimating to the similar problem for the local multiplicities and, finally, obtain the necessary estimates for the global multiplicities.

In Sec. 5.5 we complete the proof of Theorem 2 .

5.1. Tuples of polynomials with a positive-dimensional set of solutions. As always, the symbol $\mathcal{P}_{i, N}$ denotes the space of homogeneous polynomials of degree $i$ in $N$ variables, we identify $\mathcal{P}_{i, N+1}$ and $H^{0}\left(\mathbb{P}^{N}, \mathcal{O}_{\mathbb{P}^{N}}(i)\right)$. Let

$$
\mathcal{H}_{N}^{\left(n_{1}, n_{2}\right)}=\prod_{i=1}^{n_{1}} \mathcal{P}_{2, N} \times \prod_{i=1}^{n_{2}} \mathcal{P}_{3, N}
$$

be the space of all tuples $\left(f_{1}, \ldots, f_{n_{1}}, f_{n_{1}+1}, \ldots, f_{n_{1}+n_{2}}\right)$, where the first $n_{1}$ polynomials are quadratic, the next $n_{2}$ ones are cubic. (This is the global analog of the space 
$\mathcal{P}_{N}^{\left(n_{1}, n_{2}\right)}$, introduced in $\S 2$.) In this section we consider the space $\mathcal{H}_{N+1}^{\left(n_{1}, n_{2}\right)}$ for $n_{1}+n_{2}=$ $N$ and $N+1$. The symbol

$$
Z\left(f_{1}, \ldots, f_{n_{1}+n_{2}}\right)=Z\left(f_{*}\right)
$$

denotes the closed subscheme, defined by the tuple of polynomials $f_{1}, \ldots, f_{n_{1}+n_{2}}$, and the symbol $\left|Z\left(f_{*}\right)\right|$ stands for the closed set $\left\{f_{1}=\ldots=f_{n_{1}+n_{2}}=0\right\} \subset \mathbb{P}^{N}$. Let

$$
Y_{\infty}=\left\{\left(f_{1}, \ldots, f_{n_{1}+n_{2}}\right) \mid \operatorname{dim} Z\left(f_{*}\right) \geq 1\right\} \subset \mathcal{H}_{N+1}^{\left(n_{1}, n_{2}\right)}
$$

be the closed subset of tuples, the zeros of which have an "incorrect" dimension (for a generic tuple for $n_{1}+n_{2}=N$ the set $Z\left(f_{*}\right)$ is zero-dimensional, for $n_{1}+n_{2}=N+1$ it is empty). Furthermore, define $Y_{\text {line }} \subset Y_{\infty}$ as the set, consisting of such tuples $\left(f_{*}\right)$, that the set $Z\left(f_{*}\right)$ contains a line in $\mathbb{P}^{N}$. It is easy to compute that for $n_{1}+n_{2}=N$

$$
\operatorname{codim} Y_{\text {line }}=n_{1}+2 n_{2}+2,
$$

and for $n_{1}+n_{2}=N+1$

$$
\operatorname{codim} Y_{\text {line }}=n_{1}+2 n_{2}+4
$$

(the codimension is in both cases with respect to the space $\mathcal{H}_{N+1}^{\left(n_{1}, n_{2}\right)}$ ). Set $Y_{\infty}^{\prime}=$ $\overline{Y_{\infty} \backslash Y_{\text {line }}}$ to be the union of all irreducible components of the set $Y_{\infty}$, different from $Y_{\text {line. Now we have }}$

Proposition 5.1. The irreducible closed set $Y_{\text {line }}$ is a component of maximal dimension of the closed set $Y_{\infty}$ :

$$
\operatorname{dim} Y_{\text {line }} \geq \operatorname{dim} Y_{\infty}^{\prime} .
$$

Proof. The irreducibility of the set $Y_{\text {line }}$ is obvious. The claim of the proposition will be proved by the method developed in [2]. Consider first the case $n_{1}+n_{2}=N$. Let $Y_{i} \subset \mathcal{H}_{N+1}^{\left(n_{1}, n_{2}\right)}$ be the set of such tuples $\left(f_{*}\right)$, that

(1) $\operatorname{codim}\left\{f_{1}=\ldots=f_{i}=0\right\}=i$,

(2) there is a component $B$ of the set $Z\left(f_{1}, \ldots, f_{i}\right)$, which for $i=N-1$ is not a line in $\mathbb{P}^{N}$ and on which the polynomial $f_{i+1}$ vanishes identically. It is sufficient to show that

$$
\operatorname{codim} \overline{Y_{i}}>n_{1}+2 n_{2}+2
$$

for $i=1, \ldots, N-1$.

Following [2, §3], represent $Y_{i}$ as the union

$$
Y_{i}=Y_{i, 0} \cup Y_{i, 1} \cup \ldots \cup Y_{i, i}
$$

of smaller subsets $Y_{i, e}, e \in\{0, \ldots, i\}$, defined by the condition: the set $B$ has codimension $e \in \mathbb{Z}_{+}$in its linear span $\langle B>$. Let us consider, first of all, the 
case $e=0$, that is, $B \subset \mathbb{P}^{N}$ is a linear subspace, $i \leq N-2$. Since $\left.f_{j}\right|_{B} \equiv 0$ for $j=1, \ldots, i+1$ and $d_{j}=\operatorname{deg} f_{j} \geq 2$, we get the estimate

$$
\begin{gathered}
\operatorname{codim} Y_{i, 0} \geq(i+1) \frac{(N-i+1)(N-i+2)}{2}-i(N-i+1)= \\
=\frac{N-i+1}{2}(i(N-i)+N-i+2) \geq 3 N,
\end{gathered}
$$

which is what we need (the minimum of the right hand side is attained at $i=N-2$, which corresponds to the set of such tuples $\left(f_{*}\right)$ that $Z\left(f_{*}\right)$ contains a plane).

Therefore, we may assume that $e \geq 1$. In that case consider the restrictions $\left.f_{j}\right|_{<B>}, j=1, \ldots, i$. Recall the following [2]

Definition 5.1. Let $h_{1}, \ldots, h_{m}$ be homogeneous polynomials of degree $\geq 2$ on the projective space $\Pi$ of dimension $\operatorname{dim} \Pi \geq m+1$. An irreducible subvariety $C \subset \Pi$ such that $\langle C\rangle=\Pi$ and $\operatorname{codim} C=m$ is called an associated subvariety of the sequence $\left(h_{*}\right)$, if there exists a chain of irreducible subvarieties $C_{j} \subset \Pi$, $j=0, \ldots, m$, satisfying the following properties:

- $C_{0}=\Pi$,

- for each $j=0, \ldots, m-1$ the subvariety $C_{j+1}$ is an irreducible component of the closed algebraic set $\left\{h_{j+1}=0\right\} \cap C_{j}$, and moreover, $\left.h_{j+1}\right|_{C_{j}} \not \equiv 0$, so that $\operatorname{codim}_{\Pi} C_{j}=j$ for all $j$,

- $C_{m}=C$.

If a sequence $\left(h_{*}\right)$ has an associated subvariety, it is said to be good.

Furthermore, the following claim is true.

Lemma 5.1. (i) The property of being good is an open property in the space of sequences.

(ii) A good sequence $\left(h_{*}\right)$ can have, at most,

$$
\left[\frac{1}{m+1} \prod_{j=1}^{m} \operatorname{deg} h_{j}\right]
$$

associated subvarieties.

Proof. This is Lemma 4 in [2].

As it was shown in [2, p.73], one can find among the polynomials $f_{j}, j=1, \ldots, i$, such $e$ polynomials $f_{j_{1}}, \ldots, f_{j_{e}}$ that the sequence $\left(\left.f_{j_{1}}\right|_{<B>}, \ldots,\left.f_{j_{e}}\right|_{<B>}\right)$ is good and $B$ is one of its associated subvarieties. Besides, as it was shown in [2, p.72], for a fixed irreducible subvariety $C$ in the projective space $\Pi$, such that $\langle C\rangle=\Pi$, the requirement $\left.h\right|_{C} \equiv 0$ imposes on the polynomial $h$ at least

$$
\operatorname{deg} h \cdot \operatorname{dim} \Pi+1
$$


independent conditions. For that reason, fixing the subspace $<B>$ and the polynomials $f_{j_{1}}, \ldots, f_{j_{e}}$, we obtain that the requirement $\left.f_{j}\right|_{B} \equiv 0$ imposes on the polynomials $f_{j}$, where

$$
j \in\{i+1\} \cup\left(\{1, \ldots, i\} \backslash\left\{j_{1}, \ldots, j_{e}\right\}\right)
$$

at least

$$
(i-e+1)(2(N-i+e)+1)
$$

independent conditions. Subtracting the dimension of the Grassmanian of $(N-i+e)$ subspaces in $\mathbb{P}^{N}$, we get

$$
\operatorname{codim} Y_{i, e} \geq(i-e+2)(N-i+e)+1
$$

It is easy to check that the minimum of the right hand side is $2 N+1$. The inequality

$$
2 N+1 \geq n_{1}+2 n_{2}+2
$$

is always true, except for the only case $n_{1}=0, n_{2}=N$. However, estimating the number of independent conditions above, we assumed that $\operatorname{deg} f_{j}=2$. If all the polynomials are cubic ones, the estimate becomes essentially stronger:

$$
\operatorname{codim} Y_{i, e} \geq(2 i-2 e+3)(N-i+e)+1 \geq 3 N+1
$$

which completes the proof of the proposition in the case $n_{1}+n_{2}=N$.

Consider the case $n_{1}+n_{2}=N+1$. Let $\left(f_{1}, \ldots, f_{N+1}\right)$ be a tuple of general position in some irreducible component $Q$ od the set $Y_{\infty}^{\prime}$. Then there are two options: either $\operatorname{dim} Z\left(f_{1}, \ldots, f_{N}\right)=1$ and $f_{N+1}$ vanishes on some irreducible component $C$ of the set $Z\left(f_{1}, \ldots, f_{N}\right)$, and moreover the curve $C$ is not a line, or $\operatorname{dim} Z\left(f_{1}, \ldots, f_{N}\right) \geq 2$ and $f_{N+1}$ is an arbitrary polynomial.

Assume that the first option takes place. According to what was proved above, the codimension of the set

$$
\left\{\left(f_{1}, \ldots, f_{N}\right) \mid\left(f_{1}, \ldots, f_{N+1}\right) \in Q\right\} \subset \mathcal{H}_{N+1}^{\left(n_{1}, n_{2}-1\right)}
$$

is not smaller than $n_{1}+2\left(n_{2}-1\right)+2$, if $n_{1} \leq N$ (that is, if at least one polynomial is a cubic one), and $n_{1}+1$, if $n_{1}=N+1$. It is easy to see that the condition $\left.f_{N+1}\right|_{C} \equiv 0$, where $C$ is a curve, the linear span of which is of dimension at least 2 , imposes on the polynomial $f_{N+1}$ at least 5 condition. Therefore, $\operatorname{codim} Q>\operatorname{codim} Y_{\text {line }}$, as we claimed.

Assume that the second option takes place. Here we may assume that $\operatorname{dim} Z\left(f_{1}, \ldots, f_{N}\right)=$ 2. The polynomial $f_{N+1}$ is an arbitrary one, so swapping $f_{N+1}$ and some polynomial $f_{i}, i \leq N$, we get the situation considered above. Q.E.D. for proposition 5.1.

Now consider the set

$$
Y_{i}^{\Delta} \subset \mathcal{H}_{N+1}^{\left(n_{1}, n_{2}\right)} \backslash Y_{\infty}
$$

consisting of such tuples $\left(f_{1}, \ldots, f_{N+1}\right)$, that there are $i+1$ distinct points $p_{1}, \ldots, p_{i+1} \in$ $Z\left(f_{*}\right)$, such that

$$
\operatorname{dim}<p_{1}, \ldots, p_{i+1}>\leq i-1 .
$$


The following claim is true.

Proposition 5.2. The equality

$$
\operatorname{codim} Y_{i}^{\Delta}=N+2
$$

holds.

Proof. For convenience set $Y_{1}^{\Delta}=\emptyset$. It is sufficient to show that $\operatorname{codim}\left(Y_{i}^{\Delta} \backslash Y_{i-1}^{\Delta}\right)=$ $N+2$, that is, we may assume that among the points $p_{*}$ any $i$ points are linearly independent, so that $\left.\Lambda=<p_{*}\right\rangle$ is a subspace of dimension $i-1$. Let us fix $\Lambda$. It is easy to see that the linear conditions $f_{j}\left(p_{i}\right)=0$ are linearly independent, so that the set

$$
\left\{\left(f_{1}, \ldots, f_{N+1}\right)\left|\left\{p_{1}, \ldots, p_{i+1}\right\} \subset\right| Z\left(f_{*}\right) \mid\right\}
$$

has codimension $(i+1)(N+1)$. The set of tuples $\left\{p_{1}, \ldots, p_{i+1}\right\} \subset \Lambda$ has dimension $(i+1)(i-1)$, whereas the dimension of the projective Grassmanian is $i(N-i+1)$. As a result we get

$$
\operatorname{codim}\left(Y_{i}^{\Delta} \backslash Y_{i-1}^{\Delta}\right)=(i+1)(N-i+2)-i(N-i+1)=N+2,
$$

as we claimed. Q.E.D. for the proposition.

Propositions 5.1 and 5.2 immediately imply

Proposition 5.3. For $a \leq N+1$ the equality

$$
\operatorname{codim}\left\{\left(f_{1}, \ldots, f_{N+1}\right)|\sharp| Z\left(f_{*}\right) \mid \geq a\right\}=a
$$

holds.

Proof. One may assume that the set $\left|Z\left(f_{*}\right)\right|$ is finite and consists of $a$ linearly independent points. In that case the claim of the proposition is obvious. Q.E.D.

5.2. The global multiplicities: the definitions and setting up the problem. We start to globalize the local theory, developed in $\S \S 2-4$. Now, in order to distinguish between the local and global multiplicities, for the local multiplicities of the type $\mu_{l, N}(a, b ; d)$ we write $\mu_{l, N}^{\text {local }}(a, b ; d)$ etc. Consider the space

$$
\mathcal{H}_{N+1}^{\left(n_{1}, n_{2}\right)}=\left(\mathcal{P}_{2, N+1}\right)^{n_{1}} \times\left(\mathcal{P}_{3, N+1}\right)^{\times n_{2}}
$$

of tuples $\left(f_{1}, \ldots, f_{n_{1}}, f_{n_{1}+1}, \ldots, f_{n_{1}+n_{2}}\right)$, consisting of $n_{1}$ quadratic and $n_{2}$ cubic polynomials, which we see as polynomials on $\mathbb{P}^{N}$. Assume that $n_{1}+n_{2}=N+1$. Let

$$
\Sigma_{1}\left(f_{*}\right)=<f_{1}, \ldots, f_{n_{1}}>
$$

be the linear system, generated by the quadratic polynomials, and

$$
\Sigma_{2}\left(f_{*}\right)=<f_{n_{1}+1}, \ldots, f_{n_{1}+n_{2}}>+\Sigma_{1} \mathcal{P}_{1, N+1}
$$

be the linear system of cubic polynomials, generated by all polynomials $f_{*}$. 
By the symbol $Y_{\infty}$ we denote the closed subset of the space $\mathcal{H}_{N+1}^{\left(n_{1}, n_{2}\right)}$, consisting of the tuples $\left(f_{*}\right)$ with the zero set of positive dimension: $\operatorname{dim} Z\left(f_{*}\right) \geq 1$. According to Proposition 5.1, we have codim $Y_{\infty}=n_{1}+2 n_{2}+4$. Let

$$
\left(f_{1}, \ldots, f_{N+1}\right) \in \mathcal{H}_{N+1}^{\left(n_{1}, n_{2}\right)} \backslash Y_{\infty}
$$

be an arbitrary tuple.

We write $\Sigma_{1}=\Sigma_{1}\left(f_{*}\right)$ and $\Sigma_{2}=\Sigma_{2}\left(f_{*}\right)$, if it is clear, which tuple is meant. Consider the set

$$
\Sigma_{1}^{n_{1}} \times \Sigma_{2}^{N-n_{1}} \subset\left(\mathcal{P}_{2, N+1}\right)^{\times n_{1}} \times\left(\mathcal{P}_{3, N+1}\right)^{\times n_{2}}
$$

Let $\left(f_{1}^{\sharp}, \ldots, f_{N}^{\sharp}\right) \in \Sigma_{1}^{n_{1}} \times \Sigma_{2}^{N-n_{1}}$ be a tuple of general position. For an arbitrary effective cycle $R$ of pure codimension $l \in \mathbb{Z}_{+}$on $\mathbb{P}^{N}$ we define the global effective multiplicity by the equality

$$
\mu_{\text {total }}\left(\left(f_{*}\right) ; R\right)=\sum_{p \in\left|Z\left(f_{*}\right)\right|} \mu_{\text {local }}\left(\left(f_{*}\right) ; R, p\right)
$$

(recall that the set $\left|Z\left(f_{*}\right)\right|$ is finite, since the tuple $\left(f_{*}\right)$ has been chosen outside the subset $\left.Y_{\infty}\right)$, where the local multiplicity at the point $p$ is meant in the sense of Sec. 2.1:

$$
\mu_{\text {local }}\left(\left(f_{*}\right) ; R, p\right)=\operatorname{mult}_{p}\left(\left\{f_{l+1}^{\sharp}=0\right\} \circ \ldots \circ\left\{f_{N}^{\sharp}=0\right\} \circ R\right) .
$$

(Obviously, in the right hand side in brackets we have a zero-dimensional cycle, so that mult $_{p}$ is the multiplicity of the point $p$ in that cycle.) If $R \subset \mathbb{P}^{N}$ is an irreducible subvariety of codimension $l$, then

$$
\mu_{\text {local }}\left(\left(f_{*}\right) ; R, p\right)=\operatorname{dim} \mathcal{O}_{p, R} /\left(f_{l+1}^{\sharp}, \ldots, f_{N}^{\sharp}\right) .
$$

Furthermore, let $Y^{\Delta} \subset \mathcal{H}_{N+1}^{\left(n_{1}, n_{2}\right)} \backslash Y_{\infty}$ be the set of such tuples $\left(f_{*}\right)$, that in the finite set of points $\left|Z\left(f_{*}\right)\right|$ one can choose a linearly dependent subset. By Proposition 5.2 ,

$$
\operatorname{codim} \overline{Y^{\Delta}}=N+2 .
$$

Therefore, we get the presentation

$$
\mathcal{H}_{N+1}^{\left(n_{1}, n_{2}\right)} \backslash\left(Y_{\infty} \cup \bar{Y}^{\Delta}\right)=Y_{0} \sqcup Y_{1} \sqcup \ldots \sqcup Y_{N+1},
$$

where $Y_{a}$ is a constructive set, such that for every tuple $\left(f_{*}\right) \in Y_{a}$ the set of zeros $\left|Z\left(f_{*}\right)\right|$ consists of precisely $a \in\{0, \ldots, N+1\}$ linearly independent points. The closures $\overline{Y_{a}}$ are irreducible, the sets $Y_{a}$ are open in their closures and, by Proposition 5.3, codim $\overline{Y_{a}}=a$. In particular, $Y_{0} \subset \mathcal{H}_{N+1}^{\left(n_{1}, n_{2}\right)}$ is an open subset.

As in $\S 2$, the symbol $\mathbb{H}_{l, N}(d)$ stands for the Chow variety, parametrizing effective cycles of pure codimension $l \in \mathbb{Z}_{+}$and degree $d \geq 1$ on the projective space $\mathbb{P}^{N}$. 
Define the subset

$$
\mathcal{Y}_{l, N, i}^{\text {total }}(m, d) \subset Y_{i} \times \mathbb{H}_{l, N}(d)
$$

by the condition $\mu_{\text {total }}\left(\left(f_{*}\right) ; R\right) \geq m \in \mathbb{Z}_{+}$. This is a closed algebraic subset (for a given value $i \in\{0, \ldots, N+1\})$. By one and the same symbol $\pi_{\mathcal{P}}$ we denote the projection of the direct product $Y_{i} \times \mathbb{H}_{l, N}(d)$ onto $Y_{i}$. Since the Chow varieties are projective, the image

$$
Y_{l, N, i}^{\mathrm{total}}(m, d)=\pi_{\mathcal{P}}\left(\mathcal{Y}_{l, N, i}^{\mathrm{total}}(m, d)\right) \subset Y_{i}
$$

is a closed subset. It is clear that $Y_{i}$ and $Y_{l, N, i}^{\text {total }}(m, d)$ are all invariant with respect to the action of the group $G L_{N+1}(\mathbb{C})$ of linear changes of coordinates. Let us consider the problem of estimating the codimension

$$
\operatorname{codim}\left(Y_{l, N, i}^{\mathrm{total}}(m, d) \subset Y_{i}\right)
$$

Apart from the group $G L_{N+1}(\mathbb{C})$, on the space $\mathcal{H}_{N+1}^{\left(n_{1}, n_{2}\right)}$ acts the group $G^{*}\left(n_{1}, n_{2}\right)$. This action is similar to the action of the group $G\left(n_{1}, n_{2}\right)$ in the local situation. More precisely, the elements $g \in G^{*}\left(n_{1}, n_{2}\right)$ are triples

$$
g=\left(A_{11} \in G L_{n_{1}}(\mathbb{C}), A_{22} \in G L_{n_{2}}(\mathbb{C}), A_{12} \in \operatorname{Mat}_{\left(n_{1}, n_{2}\right)}\left(\mathcal{P}_{1, N+1}\right)\right),
$$

here $g\left(f_{1}, \ldots, f_{N+1}\right)=\left(f_{1}^{g}, \ldots, f_{N+1}^{g}\right)$, where

$$
\left(f_{1}^{g}, \ldots, f_{n_{1}}^{g}\right)=\left(f_{1}, \ldots, f_{n_{1}}\right) A_{11}
$$

and

$$
\left(f_{n_{1}+1}^{g}, \ldots, f_{n_{1}+n_{2}}^{g}\right)=\left(f_{n_{1}+1}, \ldots, f_{n_{1}+n_{2}}\right) A_{22}+\left(f_{1}, \ldots, f_{n_{1}}\right) A_{12}
$$

Subsets that are invariant both with respect to linear changes of coordinates and the action of the group $G^{*}\left(n_{1}, n_{2}\right)$, are, as in the local case, said to be bi-invariant.

It is obvious that all the sets $Y_{\infty}, Y^{\Delta}, Y_{i}$ and $Y_{l, N, i}^{\text {total }}(m, d)$ are bi-invariant.

By construction, the set $Y_{l, N, i}^{\mathrm{total}}(m, d)$ consists of such tuples of polynomials $\left(f_{1}, \ldots, f_{N+1}\right) \in$ $Y_{i}$, that there exists an effective cycle $R$ of pure codimension $l$ and degree $d$ on $\mathbb{P}^{N}$, satisfying the inequality $\mu_{\text {total }}\left(\left(f_{*}\right) ; R\right) \geq m$. Similar to the local case, we reformulate the problem of estimating the codimension of the set $Y_{l, N, i}^{\text {total }}(m, d)$ : we will maximize the multiplicity $m$ for a fixed codimension. More precisely, let $B \subset Y_{i}$ be a bi-invariant irreducible subvariety. Set

$$
\mu_{\text {total }}(B, d)=\max \left\{m \mid B \subset Y_{l, N, i}^{\text {total }}(m, d)\right\}
$$

(the indices $l, N$ are omitted in the left hand side to simplify the notations). Explicitly,

$$
\mu_{\text {total }}(B, d)=\max _{R}\left\{\mu_{\text {total }}\left(\left(f_{*}\right) ; R\right)\right\}
$$


the maximum is taken over all effective cycles of degree $d$ and codimension $l$, and, as usual, $\left(f_{*}\right) \in B$ is a generic tuple. Finally, set $\mu_{\text {total }}(i, a, d)=m$, if there exists an irreducible bi-invariant subvariety $B \subset Y_{i}$ of codimension at most $a$, such that $\mu_{\text {total }}(B, d)=m$, and such a variety does not exist for $m+j, j \geq 1$. Assuming the codimension $a$ to be fixed, let us estimate the multiplicity $m$ from above.

5.3. The local and global type of a subvariety of tuples of polynomials. Fix an irreducible bi-invariant subvariety $B \subset Y_{r}, r \geq 1$. For a generic (and arbitrary) tuple $\left(f_{*}\right) \in B$ the set theoretic intersection $\left|Z\left(f_{*}\right)\right|$ consists of precisely $r$ linearly independent points $p_{1}, \ldots, p_{r} \in \mathbb{P}^{N}$ (depending on the tuple $\left(f_{*}\right)$, of course). For a point $p \in\left|Z\left(f_{*}\right)\right|$ set $\varepsilon_{p}\left(f_{*}\right)=b$, where

$$
\operatorname{rk}\left(d f_{1}(p), \ldots, d f_{N+1}(p)\right)=N-b .
$$

Set $b_{i}=\varepsilon_{p_{i}}\left(f_{*}\right) \in \mathbb{Z}_{+}, i=1, \ldots, r$. Obviously, the tuple of integers $\left(b_{1}, \ldots, b_{r}\right) \in \mathbb{Z}_{+}^{r}$ does not depend on the choice of the tuple $\left(f_{*}\right)$ and makes an invariant of the subvariety $B$. We will assume that the inetegers $b_{i}$ are ordered: $b_{1} \geq b_{2} \geq \ldots \geq b_{r}$.

Definition 5.2. The ordered (non-increasing) tuple of integers $\left(b_{1}, \ldots, b_{r}\right)$ is called the global type of the bi-invariant subvariety $B \subset Y_{r}$, the notation: $\varepsilon_{\text {total }}(B)=$ $\left(b_{*}\right)$.

Set

$$
r_{*}=\max \left\{j \mid b_{j} \geq j, 1 \leq j \leq r\right\},
$$

if $b_{1} \geq 1$; if $b_{1}=\ldots=b_{r}=0$, then we set $r_{*}=0$. Set $\Phi(0, \ldots, 0)=0$, and for $b_{1} \geq 1$

$$
\Phi\left(b_{1}, \ldots, b_{r}\right)=\sum_{j=1}^{r_{*}}\left(b_{j}+1\right)\left(b_{j}+1-j\right) .
$$

Now we have

Lemma 5.2. The following estimate holds:

$$
\operatorname{codim}\left(B \subset Y_{r}\right) \geq \Phi\left(b_{1}, \ldots, b_{r}\right)
$$

Proof. If $b_{1}=\ldots=b_{r}=0$, then there is nothing to prove. So assume that $b_{1} \geq 1$. By the bi-invariance, it is sufficient to show that for fixed linearly independent points $p_{1}, \ldots, p_{r} \in \mathbb{P}^{N}$ the estimate

$$
\operatorname{codim}\left(B\left(p_{1}, \ldots, p_{r}\right) \subset Y_{r}\left(p_{1}, \ldots, p_{r}\right)\right) \geq \Phi\left(b_{*}\right)
$$

holds, where $Y_{r}\left(p_{*}\right)=\left\{\left(f_{*}\right) \in Y_{r}\left|\left\{p_{1}, \ldots, p_{r}\right\}=\right| Z\left(f_{*}\right) \mid\right\}$ и $B\left(p_{*}\right)=B \cap Y_{r}\left(p_{*}\right)$. Furthermore, taking an affine chart $\mathbb{C}_{\left(z_{1}, \ldots, z_{N}\right)}^{N} \subset \mathbb{P}^{N}$, we may assume that $p_{1}$ is the origin, and for $j \geq 2$

$$
p_{j}=(0, \ldots, 0,1,0, \ldots, 0)
$$


where the only unity occupies the $(j-1)$-th position. The closed subset $B\left(p_{*}\right) \subset$ $Y_{r}\left(p_{*}\right)$ may consist of several irreducible components. Take any component $B^{+}$of that set, the generic tuple $\left(f_{*}\right) \in B^{+}$in which satisfies the equalities

$$
\varepsilon_{p_{i}}\left(f_{*}\right)=b_{i}
$$

Obviously, the lemma will be shown if we prove the inequality

$$
\operatorname{codim}\left(B^{+} \subset Y_{r}\left(p_{*}\right)\right) \geq \Phi\left(b_{*}\right) .
$$

That is what we will do. To simplify the arguments, assume that all polynomials $f_{i}$ are quadratic: if $\operatorname{deg} f_{i}=3$, then the arguments work without modification, whereas the estimates for the codimension only get stronger (there are more coefficients).

Write down explicitly

$$
f_{i}=a_{1}^{(i)} z_{1}+\ldots+a_{N}^{(i)} z_{N}+\sum_{j \leq k} a_{j k}^{(i)} z_{j} z_{k} .
$$

If $r \geq 2$, then the condition $f_{i}\left(p_{j}\right)=0$ takes the form of the equalities

$$
a_{j}^{(i)}+a_{j j}^{(i)}=0
$$

for all $i$ and $j=1, \ldots, r-1$. By the bi-invariance, we may assume that

$$
\operatorname{rk}\left(d f_{1}(o), \ldots, d f_{N-b_{1}}(o)\right)=N-b_{1},
$$

and the linear forms $d f_{i}(o)$ are linear combinations of the first $N-b_{1}$ forms $d f_{1}(o), \ldots, d f_{N-b_{1}}(o)$ for $i \geq N-b_{1}+1$. This gives $b_{1}\left(b_{1}+1\right)$ independent conditions on the coefficients $a_{j}^{(i)}$ for $i \geq N-b_{1}+1$, assuming the polynomials $f_{1}, \ldots, f_{N-b}$ to be fixed. If $r=1$, then there is nothing more to prove.

Assume that $r \geq 2$ and consider the conditions, associated with the point $p_{2}=$ $(1,0, \ldots, 0)$. Recall that $b_{2} \leq b_{1}$. If $r_{*}=1$, there is nothing to prove. Therefore we assume that $b_{2} \geq 2$. Again we assume the polynomials $f_{1}, \ldots, f_{N-b_{2}}$ to be fixed (which does not contradict the first step of the proof above), so that the linear forms $d f_{1}\left(p_{2}\right), \ldots, d f_{N-b_{2}}\left(p_{2}\right)$ are linearly independent, and $d f_{i}\left(p_{2}\right)$ for $i \geq N-b_{2}+1$ are their linear combinations. Explicitly,

$$
d f_{i}\left(p_{2}\right)=\left(a_{1}^{(i)}+2 a_{11}^{(i)}\right) z_{1}+\sum_{j \geq 2}\left(a_{j}^{(i)}+a_{1 j}^{(i)}\right) z_{j} .
$$

By the equality $a_{1}^{(i)}=-a_{11}^{(i)}$ the coefficient at $z_{1}$ is linearly dependent on the set of coefficients $a_{j}^{(i)}$. However, the coefficients $a_{1 j}^{(i)}$ were not involved in the conditions, associated with the point $p_{1}$. Therefore, requiring that

$$
\left.d f_{i}\left(p_{2}\right)\right|_{\left\{z_{1}=0\right\}} \in<\left.d f_{1}\left(p_{2}\right)\right|_{\left\{z_{1}=0\right\}}, \ldots,\left.d f_{N-b_{2}}\left(p_{2}\right)\right|_{\left\{z_{1}=0\right\}}>
$$


for $i \geq N-b_{2}+1$, we impose at least

$$
\left(b_{2}+1\right)\left(b_{2}-1\right)
$$

independent conditions on the coefficients $a_{1 j}^{(i)}$. (The precise number of conditions is determined by the dimension of the space in the right hand side of the formula (28): if it is equal to $N-b_{2}$, then we get $\left(b_{2}+1\right)\left(b_{2}-1\right)$ conditions, if it drops by one, then we get $\left(b_{2}+1\right) b_{2}$ conditions.) This completes our consideration of the second component $(j=2)$ of the function $\Phi$. If $r_{*}=2$, our lemma is shown.

If $r_{*} \geq 3$, then we continue in the same spirit: arguing by induction, we assume that it is shown that the condition

$$
\operatorname{rk}\left(d f_{1}\left(p_{\alpha}\right), \ldots,\left(d f_{N+1}\left(p_{\alpha}\right)\right)=N-b_{\alpha}\right.
$$

for $\alpha=1, \ldots, j$ imposes on the coefficients $a_{\gamma}^{(i)}, i \geq N-b_{1}+1$, and $a_{\lambda k}^{(i)}$, where $\lambda=1, \ldots, j-1, k=\lambda+1, \ldots, N$ and $i \geq N-b_{\lambda+1}+1$, in total at least

$$
\sum_{\lambda=1}^{j}\left(b_{\lambda}+1\right)\left(b_{\lambda}+1-\lambda\right)
$$

independent conditions. If $r_{*}=j$, then the proof is complete at that step. Otherwise, assuming $f_{1}, \ldots, f_{N-b_{j+1}}$ to be fixed, we get that the linear forms

$$
\left.d f_{i}\left(p_{j+1}\right)\right|_{\left\{z_{1}=\ldots=z_{j}=0\right\}}, \quad i \geq N-b_{j+1}+1,
$$

belong to the linear space

$$
<\left.d f_{1}\left(p_{j+1}\right)\right|_{\left\{z_{1}=\ldots=z_{j}=0\right\}}, \ldots,\left.d f_{N-b_{j+1}}\left(p_{j+1}\right)\right|_{\left\{z_{1}=\ldots=z_{j}=0\right\}}>.
$$

This gives at least $\left(b_{j+1}+1\right)\left(b_{j}-j\right)$ independent conditions for the new (that is, not involved in the previous considerations) coefficients

$$
a_{j, j+1}^{(i)}, \ldots, a_{j, N}^{(i)}, \quad i \geq N-b_{j+1}+1 .
$$

Now the inductive step from $j$ to $j+1$ is constructed and the proof of the lemma is complete.

Corollary 5.1. The following estimate holds:

$$
\mu_{\text {total }}(i, a, d) \leq \sum_{\Phi\left(b_{1}, \ldots, b_{i}\right)+i \leq a} \mu_{\text {local }}\left(a-b_{i}, b_{i} ; d\right) .
$$

Proof. This follows from the previous lemma, the equality codim $Y_{i}=i$ and the fact that the global multiplicity $\mu_{\text {total }}$ is computed via the tuples of $N+1$ polynomials, whereas the local one $\mu_{\text {local }}$ via the tuples of $N$ polynomials. Indeed, if

$$
B \subset \mathcal{P}_{[1,2], N}^{\times n_{1}} \times \mathcal{P}_{[1,3], N}^{\times n_{2}}
$$


is a bi-invariant subvariety of codimension $a$ (in the sense of the local theory $\S \S 2-4$ ) and $\varepsilon(B)=b$, where $n_{1}+n_{2}=N+1$, then the projection $[B]_{N+1}$ of the set $B$ along the last direct factor has codimension at most $a-b$ in the space $\mathcal{P}_{[1,2], N}^{\times n_{1}} \times \mathcal{P}_{[1,3], N}^{\times\left(n_{2}-1\right)}$, since for a generic tuple $\left(f_{1}, \ldots, f_{N+1}\right) \in B$ we have: $d f_{N+1}(o)$ vanishes on a subspace of codimension $b$, that depends on $\left(f_{1}, \ldots, f_{N}\right)$ only. Now the claim of the corollary is obvious. Q.E.D.

To simplify the notations, we will omit the parameters $l$ and $N$, the more so that our estimates do not depend on those parameters, and write

$$
\mu_{\text {local }}(a, b ; d)
$$

instead of $\mu_{l, N}^{\text {local }}(a, b ; d)$. The values of the parameters $l$ and $N$ are in any case fixed in the subsequent arguments.

5.4. An explicit estimate for the global multiplicity. Now everything is ready to obtain an effective estimate for the global multiplicity $\mu_{\text {total }}(r, a, d)$. Let $B \subset Y_{r}$ be an irreducible bi-invariant subvariety, the codimension of which in the space $\mathcal{H}_{N+1}^{\left(n_{1}, n_{2}\right)}$ does not exceed $a$. In particular, the inequality $\Phi\left(b_{1}, \ldots, b_{r}\right)+r \leq a$ holds, where $\left(b_{1}, \ldots, b_{r}\right)=\varepsilon(B)$ is the global type of the subvariety $B$.

Proposition 5.4. The following inequality holds:

$$
\mu_{\text {total }}(B, d) \leq \sum_{i=1}^{r} \mu_{\text {local }}\left(a-b_{i}, b_{i} ; d\right) .
$$

Corollary 5.2. (i) The following inequality holds:

$$
\mu_{\text {total }}(r, a, d) \leq \max _{\Phi\left(b_{1}, \ldots, b_{r}\right)+r \leq a}\left\{\sum_{i=1}^{r} \mu_{\text {local }}\left(a-b_{i}, b_{i} ; d\right)\right\} .
$$

(ii) The following inequality holds:

$$
\mu_{\text {total }}(r, a, d) \leq d \cdot\left(\max _{\Phi\left(b_{1}, \ldots, b_{r}\right)+r \leq a}\left\{\sum_{i=1}^{r} \bar{\mu}_{\text {local }}\left(a-b_{i}, b_{i}\right)\right\}\right) .
$$

Proof of the corollary. The first inequality follows immediately from Proposition 5.4 by the definition of the numbers $\mu_{\text {total }}(r, a, d)$. The claim (ii) follows from (i), taking into account Proposition 4.1. Q.E.D. for the corollary.

Proof of Proposition 5.4. Let $\left(f_{*}\right) \in B$ be a tuple of general position, $\left\{p_{1}, \ldots, p_{r}\right\}=\left|Z\left(f_{*}\right)\right|$ its common zeros, where $b_{i}=\varepsilon_{p_{i}}\left(f_{*}\right), i=1, \ldots, r$. The inequality of Proposition 5.4 follows from the estimate

$$
\mu_{\text {local }}\left(\left(f_{*}\right) ; R, p_{i}\right) \leq \mu_{\text {local }}\left(a-b_{i}, b_{i} ; d\right)
$$


for every effective cycle $R$ of pure codimension $l$ and degree $d$. It is the last estimate that we will prove.

Set $p=p_{i}$ and let $B(p) \subset B$ be the closed (in $B$ ) subset of tuples $\left(g_{*}\right)$, vanishing at the point $p$. By the bi-invariance of the set $B$ the original tuple $\left(f_{*}\right)$, fixed at the beginning of the proof, is a generic tuple of one of the irreducible components $B^{+}$of the set $B(p)$, and moreover, the codimension of $B^{+}$in the space $\mathcal{P}_{N}^{\left(n_{1}, n_{2}\right)}$ coincides with $\operatorname{codim}\left(B \subset \mathcal{H}_{N+1}^{\left(n_{1}, n_{2}\right)}\right)$ and for that reason does not exceed $a$ (we used the natural identification of the linear space of tuples $\left(g_{*}\right) \in \mathcal{H}_{N+1}^{\left(n_{1}, n_{2}\right)}$, vanishing at the point $p$, with the space $\mathcal{P}_{N}^{\left(n_{1}, n_{2}\right)}$, defined in 2$)$. Let

$$
\pi: \mathcal{P}_{N}^{\left(n_{1}, n_{2}\right)} \rightarrow \mathcal{P}_{N}^{\left(n_{1}, n_{2}-1\right)}
$$

be the projection along the last direct factor and $\left[B^{+}\right]_{N}$ the closure of the image $\pi\left(B^{+}\right)$. By the bi-invariance and the condition $\varepsilon_{p}\left(f_{*}\right)=b$, the generic fibre of the projection

$$
\pi_{B}: B^{+} \rightarrow\left[B^{+}\right]_{N}
$$

has in $\mathcal{P}_{[1,3], N}$ codimension at least $b\left(d f_{N+1}(p)\right.$ vanishes on a $b$-dimensional linear subspace that depends on $d f_{1}, \ldots, d f_{N}$ only). Therefore, the inequality

$$
\operatorname{codim}\left(\left[B^{+}\right]_{N} \subset \mathcal{P}_{N}^{\left(n_{1}, n_{2}-1\right)}\right) \leq a-b
$$

holds, so that the inequality (29) is shown. Q.E.D. for Proposition 5.4.

Set

$$
\mu_{\text {total }}(a, d)=\max _{1 \leq r \leq \min \{a, N+1\}}\left\{\mu_{\text {total }}(r, a, d)\right\} .
$$

Proposition 5.5. For $a \geq 12$ the following estimate holds:

$$
\mu_{\text {total }}(a, d) \leq 3 \cdot 2^{a-6} d
$$

Proof. Assume first that $a \geq 21$. Since $r \leq a$, from the claim (i) of Corollary 5.2 and the inequality (i) of Corollary 4.3 we get

$$
\mu_{\text {total }}(a, d) \leq a \frac{e^{2}}{2 \pi[\sqrt{a}]}\left(\frac{5}{3}\right)^{[\sqrt{a}]} d .
$$

It is easy to check that for $a \geq 21$ the right hand side of the last inequality is strictly smaller than $3 \cdot 2^{a-6}$. This method, however, is very coarse. A mush more precise estimate is given by the claim (ii) of corollary 5.2. Set

$$
\bar{\mu}_{\text {total }}(a)=\max _{1 \leq r \leq a}\left(\max _{\Phi\left(b_{*}\right)+r \leq a}\left\{\sum_{i=1}^{r} \bar{\mu}\left(a-b_{i}, b_{i}\right)\right\}\right) .
$$


According to Corollary 5.2, (ii), and Proposition 4.1 the inequality

$$
\mu_{\text {total }}(a, d) \leq d \bar{\mu}_{\text {total }}(a)
$$

holds, so that to complete the proof of Proposition 5.5, it is sufficient to check that for $12 \leq a \leq 20$ the inequality $\bar{\mu}_{\text {total }}(a) \leq 3 \cdot 2^{a-6}$ holds. For small values of the codimension $a$ the function $\bar{\mu}_{\text {total }}(a)$ is easy to compute explicitly, and the results (for $1 \leq a \leq 36$ ) are given below in the table, which is organized in the following way. Each row corresponds to a certain value of $a$, which is given in the first column. In the second column of the same row we give the values of the parameters $r$ (the number of points) and $b_{1}, \ldots, b_{r}$, for which the maximum in the definition of the function $\bar{\mu}_{\text {total }}$ is attained. In the third column we give the very value of $\bar{\mu}_{\text {total }}(a)$.

\begin{tabular}{|l|l|l|}
\hline$a$ & & $\bar{\mu}_{\text {total }}(a)$ \\
\hline 1 & $r=1, b_{1}=0$ & 1 \\
\hline 2 & $r=2, b_{1}=b_{2}=0$ & 2 \\
\hline 3 & $r=3, b_{1}=b_{2}=b_{3}=0$ & 3 \\
\hline 4 & $r=4, b_{1}=b_{2}=1$ & 6 \\
\hline 5 & $r=3, b_{1}=b_{2}=b_{3}=1$ & 15 \\
\hline 6 & $r=4, b_{1}=\ldots=b_{4}=1$ & 24 \\
\hline 7 & $r=5, b_{1}=\ldots=b_{5}=1$ & 35 \\
\hline 8 & $r=6, b_{1}=\ldots=b_{6}=1$ & 48 \\
\hline 9 & $r=7, b_{1}=\ldots=b_{7}=1$ & 63 \\
\hline 10 & $r=8, b_{1}=\ldots=b_{8}=1$ & 80 \\
\hline 11 & $r=9, b_{1}=\ldots=b_{9}=1$ & 99 \\
\hline 12 & $r=10, b_{1}=\ldots=b_{10}=1$ & 120 \\
\hline 13 & $r=11, b_{1}=\ldots=b_{11}=1$ & 143 \\
\hline 14 & $r=12, b_{1}=\ldots=b_{12}=1$ & 168 \\
\hline 15 & $r=13, b_{1}=\ldots=b_{13}=1$ & 195 \\
\hline 16 & $r=7, b_{1}=\ldots=b_{7}=2$ & 308 \\
\hline 17 & $r=8, b_{1}=\ldots=b_{8}=2$ & 408 \\
\hline 18 & $r=9, b_{1}=\ldots=b_{9}=2$ & 522 \\
\hline 19 & $r=10, b_{1}=\ldots=b_{10}=2$ & 660 \\
\hline 20 & $r=11, b_{1}=\ldots=b_{11}=2$ & 814 \\
\hline 21 & $r=12, b_{1}=\ldots=b_{12}=2$ & 996 \\
\hline 22 & $r=13, b_{1}=\ldots=b_{13}=2$ & 1196 \\
\hline 23 & $r=14, b_{1}=\ldots=b_{14}=2$ & 1428 \\
\hline 24 & $r=15, b_{1}=\ldots=b_{15}=2$ & 1680 \\
\hline 25 & $r=16, b_{1}=\ldots=b_{16}=2$ & 1968 \\
\hline 26 & $r=17, b_{1}=\ldots=b_{17}=2$ & 2278 \\
\hline 27 & $r=18, b_{1}=\ldots=b_{18}=2$ & 2628 \\
\hline
\end{tabular}

The subsequent values of the function $\bar{\mu}_{\text {total }}$ are as follows:

$$
\bar{\mu}_{\text {total }}(28)=3002, \quad \bar{\mu}_{\text {total }}(29)=3420, \quad \bar{\mu}_{\text {total }}(30)=3864,
$$




$$
\begin{gathered}
\bar{\mu}_{\text {total }}(31)=4356, \quad \bar{\mu}_{\text {total }}(32)=4876, \quad \bar{\mu}_{\text {total }}(33)=5448, \\
\bar{\mu}_{\text {total }}(34)=6050, \quad \bar{\mu}_{\text {total }}(35)=6708 .
\end{gathered}
$$

All these values are attained for $r=a-9, b_{1}=\ldots=b_{r}=2$. For $a=36$ the next jump takes place: the maximum is attained for $r=12$ and $b_{1}=\ldots=b_{12}=3$ and equal to $\bar{\mu}_{\text {total }}(36)=7980$.

According to the table given above, starting from $a=12$ the required inequality $\bar{\mu}_{\text {total }}(a) \leq 3 \cdot 2^{a-6}$ holds.

This proves the proposition. Q.E.D.

Finally, from the values of the function $\bar{\mu}_{\text {total }}$, given in the table, by elementary arithmetic we get

Proposition 5.6. If the triple $\left(a, n_{1}, n_{2}\right)$ is one of the following triples:

$$
(11,5,3), \quad(11,3,4), \quad(11,1,5), \quad(10,2,4), \quad(10,0,5)
$$

then the following inequality holds:

$$
\bar{\mu}_{\text {total }}(a, d) \leq 2^{n_{1}+n_{2}-4} 3^{n_{2}-1} d .
$$

5.5. Regular complete intersections. Finally, let us prove Theorem 2. Let

$$
\mathcal{B} \subset \mathbb{P} \times\left(\mathcal{P}_{2, M+k+1}^{\times k_{1}} \times \mathcal{P}_{3, M+k+1}^{\times k_{2}}\right)
$$

be the closed set of "bad" pairs $\left(o,\left(f_{*}\right)\right)$, where $f_{1}(o)=\ldots=f_{k}(o)=0$ and at least one of the conditions (R1-R3) is violated at this point. Let $\pi_{1}$ and $\pi_{2}$ be the projections of the direct product on the first $(\mathbb{P})$ and second (the space of tuples of $k$ polynomials) factors, respectively. Set $\mathcal{B}(o)=\pi_{1}^{-1}(o) \cap \mathcal{B}$. The closed subset $\mathcal{B}(o)$ is contained in the subspace

$$
\mathcal{L}(o)=\mathcal{P}_{[1,2], M+k}^{\times k_{1}} \times \mathcal{P}_{[1,3], M+k}^{\times k_{2}} \subset \pi_{1}^{-1}(o)
$$

(where we again identify homogeneous polynomials vanishing at the point $o$, with non-homogeneous polynomials without the free term), and it is sufficient to show that its codimension with respect to that subspace is at least $M+1$. Indeed, if this is the case, then

$$
\operatorname{codim} \mathcal{B}=\operatorname{codim}\left(\mathcal{B}(o) \subset \pi_{1}^{-1}(o)\right) \geq M+k+1
$$

so that the map $\left.\pi_{2}\right|_{\mathcal{B}}$ can not be surjective, which immediately implies the claim of Theorem 2.

For the conditions (R1) and (R2) the inequality

$$
\operatorname{codim}(\mathcal{B}(o) \subset \mathcal{L}(o)) \geq M+1
$$


is shown in [2], taking into account Proposition 5.2. Thus it is sufficient to prove the inequality (30) for such tuples $\left(f_{*}\right) \in \mathcal{B}(o)$, which satisfy the conditions (R1) and (R2), but not the condition (R3). Fixing the linear parts of the polynomials $f_{i}$ at the point $o$, and thus the projectivized tangent space $\mathbb{T} \cong \mathbb{P}^{M-1}$, we reduce the problem to estimating the codimension of the set of tuples of $n_{1}=k_{1}+k_{2}=k$ quadratic and $n_{2}=k_{2}$ cubic homogeneous polynomials

$$
\left(\bar{q}_{1,2}, \ldots, \bar{q}_{k, 2}, \bar{q}_{k_{1}+1,3}, \ldots, \bar{q}_{k, 3}\right),
$$

for which the condition (R3) is not satisfied. However, by Propositions 5.5 and 5.6 the main inequality (3) of the condition (R3) is satisfied for a generic element of every subvariety of codimension $\leq M$ in the space of tuples

$$
\mathcal{P}_{2, M}^{\times n_{1}} \times \mathcal{P}_{3, M}^{\times n_{2}}
$$

and every irreducible subvariety $R \subset \mathbb{T}$ of codimension three. Therefore, the closed set of tuples (31), not satisfying the condition (R3), is of codimension at least $M+1$. This proves the estimate (30) and Theorem 2 as well. Q.E.D.

\section{Список литературы}

[1] Pukhlikov A. V., Birationally rigid varieties. I. Fano varieties. Russian Math. Surveys. 62 (2007), No. 5, 857-942.

[2] Pukhlikov A. V., Birationally rigid Fano complete intersections, Crelle J. für die reine und angew. Math. 541 (2001), 55-79.

[3] Pukhlikov A. V., Birationally rigid Fano complete intersections. II. arXiv:1110.2052 (math.AG), 10 p.

[4] Pukhlikov A. V., Birational automorphisms of Fano hypersurfaces, Invent. Math. 134 (1998), no. 2, 401-426.

[5] Pukhlikov A. V., Birationally rigid iterated Fano double covers. Izvestiya: Mathematics. 67 (2003), No. 3, 555-596.

[6] Fano G., Nuove ricerche sulle varieta algebriche a tre dimensioni a curve-sezioni canoniche, Comm. Rent. Ac. Sci. 11 (1947), 635-720.

[7] Iskovskikh V. A., Birational automorphisms of three-dimensional algebraic varieties, J. Soviet Math. 13 (1980), 815-868.

[8] Iskovskikh V. A. and Manin Yu. I., Three-dimensional quartics and counterexamples to the Lüroth problem, Math. USSR Sb. 86 (1971), no. 1, 140-166. 
[9] Iskovskikh V. A., Birational automorphisms of the Fano 3-fold $V_{6}^{3}$. Dokl. Akad. Nauk SSSR 235 (1977), No. 3, 509-511.

[10] Pukhlikov A. V., Maximal singularities on the Fano variety $V_{6}^{3}$. Moscow Univ. Math. Bull. 44 (1989), no. 2, 70-75.

[11] Iskovskikh V. A. and Pukhlikov A. V., Birational automorphisms of multidimensional algebraic varieties, J. Math. Sci. 82 (1996), 3528-3613.

[12] Tyurin A. N. The intersection of quadrics. Russian Math. Surveys 30 (1975), No. 6, 51-99.

[13] Tyurin A. N., Intermediate Jacobian of three-dimensional varieties. Cont. Probl. Math. 12 (1979), 5-57, 239 (loose errata), VINITI, Moscow, 1979.

[14] Pukhlikov A. V., Birational isomorphisms of four-dimensional quintics, Invent. Math. 87 (1987), 303-329.

[15] Pukhlikov A. V., Birational automorphisms of a double space and a double quadric, Math. USSR Izv. 32 (1989), 233-243.

[16] Cheltsov I. A., Non-rationality of a four-dimensional smooth complete intersection of a quadric and a quartic, not containing a plane, Sbornik: Mathematics, 194 (2003), 1679-1699.

[17] Pukhlikov A. V., Birational geometry of Fano double spaces of index two. Izvestiya: Mathematics, 74 (2010), No. 5, 925 - 991.

[18] Kollár J., et al., Flips and Abundance for Algebraic Threefolds, Asterisque 211, 1993.

[19] Pukhlikov A. V., Birationally rigid Fano varieties. In: The Fano conference, Univ. Torino, Turin, 2004, 659-681.

[20] Pukhlikov A. V. Birational geometry of algebraic varieties with a pencil of Fano complete intersections, Manuscripta Mathematica. 121 (2006), 491-526.

[21] Pukhlikov A. V., Birational geometry of algebraic varieties with a pencil of Fano cyclic covers. Pure and Appl. Math. Quart. 5 (2009), No. 2, 641-700.

[22] Gabrielov A. and Khovanskii A. G., Multiplicity of a Noetherian intersection. In: Geometry of Differential Equations. AMS, 1998, p.119-131.

[23] Pukhlikov A. V., On the multiplicity of solutions of a system of algebraic equations. Proc. Steklov Inst. Math. 276 (2012), 234-249.

[24] Fulton W., Intersection Theory, Springer-Verlag, 1984.

Department of Mathematical Sciences, The University of Liverpool

pukh@liv.ac.uk 